\title{
Measurements of the Volume Scattering Function and the Degree of Linear Polarization of Light Scattered by Contrasting Natural Assemblages of Marine Particles
}

\author{
Daniel Koestner*, Dariusz Stramski ${ }^{\circledR}$ and Rick A. Reynolds
}

Marine Physical Laboratory, Scripps Institution of Oceanography, University of California San Diego, La Jolla, CA 92093-0238, USA; dstramski@ucsd.edu (D.S.); rreynolds@ucsd.edu (R.A.R.)

* Correspondence: dkoestne@ucsd.edu; Tel: +1-631-357-9656

Received: 13 August 2018; Accepted: 13 November 2018; Published: 19 December 2018

check for updates

\begin{abstract}
The light scattering properties of seawater play important roles in radiative transfer in the ocean and optically-based methods for characterizing marine suspended particles from in situ and remote sensing measurements. The recently commercialized LISST-VSF instrument is capable of providing in situ or laboratory measurements of the volume scattering function, $\beta_{p}(\psi)$, and the degree of linear polarization, $\operatorname{DoLP}_{p}(\psi)$, associated with particle scattering. These optical quantities of natural particle assemblages have not been measured routinely in past studies. To fully realize the potential of LISST-VSF measurements, we evaluated instrument performance, and developed calibration correction functions from laboratory measurements and Mie scattering calculations for standard polystyrene beads suspended in water. The correction functions were validated with independent measurements. The improved LISST-VSF protocol was applied to measurements of $\beta_{p}(\psi)$ and $\operatorname{DoLP}_{p}(\psi)$ taken on 17 natural seawater samples from coastal and offshore marine environments characterized by contrasting assemblages of suspended particles. Both $\beta_{p}(\psi)$ and $\operatorname{DoLP}_{p}(\psi)$ exhibited significant variations related to a broad range of composition and size distribution of particulate assemblages. For example, negative relational trends were observed between the particulate backscattering ratio derived from $\beta_{p}(\psi)$ and increasing proportions of organic particles or phytoplankton in the particulate assemblage. Our results also suggest a potential trend between the maximum values of $\operatorname{DoLP}_{p}(\psi)$ and particle size metrics, such that a decrease in the maximum $\operatorname{DoLP}_{p}(\psi)$ tends to be associated with particulate assemblages exhibiting a higher proportion of large-sized particles. Such results have the potential to advance optically-based applications that rely on an understanding of relationships between light scattering and particle properties of natural particulate assemblages.
\end{abstract}

Keywords: marine optics; inherent optical properties; volume scattering function; degree of linear polarization; marine particles; light scattering measurements; LISST-VSF instrument

\section{Introduction}

It has long been recognized that inherent light-scattering properties of natural waters are of crucial importance and have strong potential for wide-ranging applications in aquatic sciences, including oceanography. These properties are essential inputs to the radiative transfer models used to compute the ambient light fields in natural water bodies [1-4]. The variability in the light scattering properties of seawater is driven primarily by the concentration of suspended particles, particle size distribution, and composition through particle refractive index, internal structure, and shape. Hence, scattering measurements carry potentially useful information about characteristics of natural particle 
assemblages. For example, the scattering and backscattering coefficients of suspended particles have been shown to provide useful proxies of mass concentration of total suspended particulate matter (SPM), particulate inorganic carbon (PIC), and particulate organic carbon (POC) in the ocean [5-7]. Multi-angle light scattering measurements provide a means to estimate the particle size distribution [8-11], including the submicrometer size range [12-14]. The angular pattern of light scattering can also contain useful information about the composition of particulate assemblages, including the bulk refractive index of particles [15-19]. In addition, measurements of the scattering matrix that provide information about polarization effects of light scattering [15,20-23] have the potential for identifying and discriminating different types of particles, such as phytoplankton species or minerals, which are present in complex natural assemblages [24-34]. Despite the potential usefulness of information provided by light scattering measurements, the complexity and variability in composition of natural particulate assemblages impose significant challenges in achieving an understanding of bulk light-scattering properties of seawater in terms of detailed compositional characteristics of particulate matter [35].

The volume scattering function, $\beta_{p}(\psi, \lambda)$, and the degree of linear polarization, $\operatorname{DoLP}_{p}(\psi, \lambda)$ of light scattered by marine particles are of primary interest in this study. Here, $\psi$ denotes the scattering angle, $\lambda$ the light wavelength in vacuum, and the subscript $p$ indicates that the quantity is associated with particles. When the subscript $p$ is omitted, the quantity describes the scattering by the entire suspension with additive contributions from both water molecules and suspended particles. The volume scattering function, $\beta(\psi, \lambda)$ [in units of $\mathrm{m}^{-1} \mathrm{sr}^{-1}$ ], is one of the fundamental inherent optical properties (IOPs) of seawater, which describes the scattered intensity as a function of scattering angle per unit incident irradiance per unit volume of small sample of water [2]. Several light-scattering related IOPs can be derived from $\beta(\psi, \lambda)$. For example, integrating $\beta(\psi, \lambda)$ over all scattering directions gives the total spectral scattering coefficient, $b(\lambda)\left[\mathrm{m}^{-1}\right]$. In this integration, it is commonly assumed that light scattering by an assemblage of randomly-oriented scatterers (molecules and particles) in natural waters is azimuthally symmetric about the incident direction of light beam. When $\beta(\psi, \lambda)$ is normalized by $b(\lambda)$, the resulting scattering phase function $\widetilde{\beta}(\psi, \lambda)\left[\mathrm{sr}^{-1}\right]$ provides a useful indicator of the angular shape of the volume scattering function. In optical remote sensing applications based on measurements with above-water sensors (e.g., from satellites or aircraft), the spectral backscattering coefficient, $b_{b}(\lambda)\left[\mathrm{m}^{-1}\right]$, is particularly useful. This coefficient can be obtained by integrating $\beta(\psi, \lambda)$ over the range of backward scattering angles [2].

The volume scattering function provides incomplete information, in the sense that it does not contain information about polarization effects associated with light scattering. A complete characterization of elastic incoherent interactions of light at arbitrary wavelength $\lambda$ with a sample volume of seawater is provided by a $4 \times 4$ scattering matrix, often referred to as the phase matrix or Mueller matrix [20-23]. This matrix describes a linear transformation of irradiance and polarization of an incident beam described by a 4-component Stokes vector into the intensity and polarization of the scattered beam that is also described by its corresponding Stokes vector. $\beta(\psi, \lambda)$ is related to the first element of the scattering matrix, $p_{11}(\psi, \lambda)$, and can be obtained from a measurement using unpolarized light for illumination of sample and measuring the total scattered intensity. The degree of linear polarization of scattered light, $\operatorname{DoL} P(\psi, \lambda)$, describes the proportion of linearly polarized light relative to total intensity of the scattered light beam. As described in greater detail below, for various assemblages of particles including suspended marine particles and when the incident light beam is unpolarized, this quantity can be derived from the first two elements of the scattering matrix, which requires measurements involving linear polarization $[29,36,37]$.

Despite the relative importance of $\beta(\psi, \lambda)$ and $\operatorname{DoLP}(\psi, \lambda)$ of seawater and the associated particulate components $\beta_{p}(\psi, \lambda)$ and $\operatorname{DoLP}_{p}(\psi, \lambda)$, the ocean optics community has historically relied mostly on simplified theoretical models (such as Mie scattering theory for homogenous spheres) and a limited dataset of measurements made with custom-built light scattering instruments. For example, over the past several decades, a limited dataset of $\beta(\psi, \lambda)$ measurements made by Petzold [38] was 
widely used as a standard input for the particulate scattering phase function for radiative transfer modeling in the ocean. Comprehensive determinations of the scattering matrix for natural seawater have been very scarce [39-42]. These determinations showed that the off-diagonal matrix elements for seawater are very small or negligible, indicating very small effects associated with optical activity or orientational anisotropy of seawater scatterers [37,42]. More recently, several light scattering sensors have been developed for in situ deployments or laboratory use [43-46], but to our knowledge, none of these sensors are commercially available. While measurements with these new sensors have already significantly contributed to the increase of available datasets of $\beta(\psi, \lambda)$ (or $\beta_{p}(\psi, \lambda)$ which can usually be satisfactorily estimated by subtracting the contribution associated with water molecules) in various oceanic environments [44,47,48], the determinations of $\operatorname{DoLP}_{p}(\psi, \lambda)$ for natural assemblages of marine particles remain very scarce, as indicated by the rarity of scattering matrix measurements of seawater.

Recently, a new light scattering instrument, the LISST-VSF (Sequoia Scientific, Inc., Bellevue, WA, USA), has become commercially available, and provides the capability of determining both the volume scattering function and the degree of linear polarization of scattered light at a single light wavelength $(532 \mathrm{~nm})$ with high angular resolution over the range $\sim 0.1^{\circ}$ to $155^{\circ}$ [49]. It is capable of both in situ and benchtop measurements on water samples. This commercial instrument is expected to enable routine measurements by different groups of investigators, so it has the potential to enhance our understanding of light scattering properties of seawater and marine particles and advance the related applications. In this study, we report on LISST-VSF measurements of $\beta_{p}(\psi)$ and $\operatorname{DoLP}_{p}(\psi)$ and size and compositional characteristics for contrasting natural particulate assemblages from marine coastal and offshore environments. The particulate scattering $\left(b_{p}\right)$ and backscattering $\left(b_{b p}\right)$ coefficients have also been determined from measured $\beta_{p}(\psi)$.

To fully realize the potential of such quantitative determinations for seawater samples from this new instrument, we also conducted an evaluation of the LISST-VSF performance through a series of laboratory experiments using samples of National Institute of Standards and Technology (NIST) certified standard polystyrene beads ranging in diameter between $100 \mathrm{~nm}$ and $2 \mu \mathrm{m}$. These measurements were compared with theoretical simulations of light scattering by bead suspensions using Mie scattering computations. With this approach, we developed corrections to the determinations of $\beta_{p}(\psi)$ and $\operatorname{DoLP} p(\psi)$ from LISST-VSF measurements. A validation of the corrected measurements was performed using independent measurements of multi-angle light scattering with another instrument, the DAWN-EOS (Wyatt Technology Corporation, Santa Barbara, CA, USA).

\section{Methods}

The description of methods includes two main parts: first, a description of laboratory experiments and Mie scattering calculations for standard polystyrene beads which were carried out to evaluate the performance of the LISST-VSF instrument and develop a calibration correction; second, a description of measurements on natural assemblages of marine particles from coastal and offshore oceanic environments.

\subsection{Laboratory Experiments and Mie Scattering Calculations to Evaluate LISST-VSF}

In order to evaluate the LISST-VSF instrument, light scattering and beam attenuation measurements were made in the laboratory on samples of nearly monodisperse standard polystyrene spherical beads with mean nominal diameters of 100, 200, 400, 500, 700, and $2000 \mathrm{~nm}$, which were suspended in water (Table 1). In addition to LISST-VSF, two other instruments were used in these experiments, a DAWN-EOS for measuring multi-angle light scattering and a dual beam UV/VIS spectrophotometer Lambda 18 (Perkin-Elmer, Inc., Waltham, MA, USA) equipped with a $15-\mathrm{cm}$ integrating sphere (Labsphere, Inc., North Sutton, NH, USA) for measuring the beam attenuation coefficient of particles in suspension. The use of standard beads ensures that Mie scattering calculations for homogeneous spherical particles can be used to calculate the Mueller matrix elements for these particles to determine reference (expected) values of the volume scattering function and the degree of 
linear polarization. The comparison of measurements with such reference values allows for evaluation of performance of LISST-VSF instrument and formulation of calibration correction functions for improved determinations of the volume scattering function and the degree of linear polarization from this instrument. This type of approach, which combines measurements on standard well-characterized particles with accurate scattering calculations, has been previously used for the evaluation, calibration, and characterization of light scattering instruments $[29,43,44,50]$. Although the evaluation results presented in this study are relevant to the specific version of the LISST-VSF instrument used in our laboratory, most methodological aspects are generally applicable to evaluation of other light scattering instruments.

Table 1. Information on the polystyrene bead size standards used to create laboratory sample suspensions for experiments. The nominal bead diameter $(D)$, catalog number, and actual mean diameter $\bar{D}$ ( \pm standard error of estimate) and standard deviation of the mean $(S D)$ provided by the manufacturer (Thermo Fisher Scientific, Inc.) is listed. The particulate beam attenuation coefficient at light wavelength $532 \mathrm{~nm}$ of the master sample as determined with a spectrophotometer, $c_{p}^{S P E C}$, is listed in addition to specific dilution names and factors (e.g., DF1, DF2, etc.) of the master suspension used for LISST-VSF measurements at different PMT gain settings. The dilution factors in italic font denote the experimental data used for generation of the final correction functions $C F_{f}$ and $B F_{f}$, and those in boldface font denote the six examples used for statistical evaluation in Table 2.

\begin{tabular}{|c|c|c|c|c|c|c|}
\hline $\begin{array}{l}\text { Nominal } D \\
{[\mathrm{~nm}]}\end{array}$ & $\begin{array}{l}\text { Catalog } \\
\text { No. }\end{array}$ & $\begin{array}{c}\bar{D} \\
{[\mathrm{~nm}]}\end{array}$ & $\begin{array}{c}S D \\
{[\mathrm{~nm}]}\end{array}$ & $\begin{array}{l}c_{p}^{S P E C} \\
{\left[\mathrm{~m}^{-1}\right]}\end{array}$ & $\begin{array}{l}\text { Dilution Factor } \\
\text { (PMT 500) }\end{array}$ & $\begin{array}{l}\text { Dilution Factor } \\
\text { (PMT 550) }\end{array}$ \\
\hline 100 & $3100 \mathrm{~A}$ & $100 \pm 3$ & 7.8 & 58.63 & $\begin{array}{c}\text { DF1: } 96, \text { DF2: } 48.5, \\
\text { DF3: } 32.7\end{array}$ & $\begin{array}{c}\text { DF1: } 96, \text { DF2: } 48.5 \text {, } \\
\text { DF3: } 32.67\end{array}$ \\
\hline 200 & $3200 \mathrm{~A}$ & $203 \pm 5$ & 5.3 & 46.26 & $\begin{array}{c}\text { DF1: } 96, D F 2: 48.5, \\
\text { DF3: } 32.7\end{array}$ & $\begin{array}{c}\text { DF1: } 96, D F 2: 48.5, \\
\text { DF3: } 32.7\end{array}$ \\
\hline 400 & $3400 \mathrm{~A}$ & $400 \pm 9$ & 7.3 & 51.44 & $\begin{array}{c}\text { DF1: } 87.4, \text { DF2: } 44.2 \\
\text { DF3: } 29.8\end{array}$ & DF1: 87.4 \\
\hline 500 & $3500 \mathrm{~A}$ & $508 \pm 8$ & 8.5 & 20.64 & DF2: 20 & \\
\hline 700 & $3700 \mathrm{~A}$ & $707 \pm 9$ & 8.3 & 50.93 & DF1: 96, DF2: 48.5 & \\
\hline 2000 & $4202 \mathrm{~A}$ & $2020 \pm 15$ & 21 & 18.21 & DF2: 20 & \\
\hline
\end{tabular}

\subsubsection{Instrumentation}

A LISST-VSF instrument (S/N 1475) was equipped with a custom designed 2 L sample chamber for benchtop laboratory use. This chamber effectively rejects ambient light and promotes good mixing conditions to maintain particles in suspension. For sample illumination the LISST-VSF uses a frequency-doubled YAG laser to produce a beam of light at a wavelength of $532 \mathrm{~nm}$ with a Gaussian beam profile of $3 \mathrm{~mm}$ in diameter. A single measurement takes approximately $4 \mathrm{~s}$ and consists of two scans of a 15-cm path within the sample, each with a different linear polarization state of the incident beam, i.e., parallel and perpendicular to the scattering plane. Scattered intensity is measured at multiple scattering angles $\psi$ from $0.09^{\circ}$ to $15.17^{\circ}$ with 32 logarithmically-spaced ring detectors and from $14^{\circ}$ to $155^{\circ}$ with $1^{\circ}$ interval using a fixed axis Roving Eyeball sensor equipped with photomultiplier tubes (PMTs). For the Roving Eyeball, scattered light is split between two PMTs with a polarizing prism allowing for only parallel or perpendicularly polarized light to be detected by each PMT. To enable measurements of large dynamic range of scattered intensity with a single PMT, the laser power is dimmed by a factor of 8 for the angular range $14-63^{\circ}$ and returned to full power for $64-155^{\circ}$. The beam attenuation coefficient, $c$, is also measured at light wavelength of $532 \mathrm{~nm}$ for the $15-\mathrm{cm}$ path length of the sample. 
For incoherent elastic scattering of light at a given wavelength $\lambda$ by a collection of particles suspended in water, the Stokes vector of incident light beam, $S_{i}=\left[I_{i} Q_{i} U_{i} V_{i}\right]^{T}$, where T represents the transpose operation, is transformed into the Stokes vector of scattered beam, $S_{s}(\psi)$, by a scattering matrix, $P(\psi)$. For an ensemble of randomly-oriented particles exhibiting certain symmetry properties and no optical activity, the scattering matrix simplifies to 6 independent non-zero elements $[20,36,51]$

$$
S_{s}(\psi)=\left[\begin{array}{c}
I_{s}(\psi) \\
Q_{s}(\psi) \\
U_{s}(\psi) \\
V_{s}(\psi)
\end{array}\right]=P(\psi) S_{i}=C\left[\begin{array}{cccc}
p_{11}(\psi) & p_{12}(\psi) & 0 & 0 \\
p_{12}(\psi) & p_{22}(\psi) & 0 & 0 \\
0 & 0 & p_{33}(\psi) & p_{34}(\psi) \\
0 & 0 & -p_{34}(\psi) & p_{44}(\psi)
\end{array}\right]\left[\begin{array}{c}
I_{i} \\
Q_{i} \\
U_{i} \\
V_{i}
\end{array}\right]
$$

where $\lambda$ has been omitted for brevity, $C$ is a constant factor (for a given sample, light wavelength, and measurement geometry), $p_{11}(\psi)$ represents the scattering phase function, and the reference plane is the scattering plane containing the incident and scattered directions $[20,51,52]$. This form provides a reasonable description of the measured scattering matrix by suspensions of randomly-oriented marine particles, including various specific types of particles present in seawater $[24,29,33,36,37,42]$. In the case of unpolarized incident light (i.e., $Q_{i}, U_{i}$, and $V_{i}$ are all zero), the volume scattering function $\beta(\psi)$ equals (to within a constant factor) $p_{11}(\psi)$, and the degree of linear polarization $\operatorname{DoLP}(\psi)$ can be determined from $[29,36,37,53]$

$$
\operatorname{DoLP}(\psi)=\frac{-p_{12}(\psi)}{p_{11}(\psi)}=\frac{-Q_{s}(\psi)}{I_{s}(\psi)} .
$$

Positive values of $\operatorname{DoLP}(\psi)$ are for dominantly perpendicular polarization and negative values for dominantly parallel polarization. We note that this definition of $\operatorname{DoLP}(\psi)$ has been widely used for characterizing the inherent scattering properties of various types of particles beyond aquatic particles, such as aerosol particles and cosmic dust [30,54-58].

The LISST-VSF measurements of forward scattering within the angular range $0.09-15.17^{\circ}$ are made with two linear polarization states of the incident beam, but with no polarization analyzers of the ring detectors. For the ring detectors, the calibrated $\beta(\psi)$ in absolute units is a standard output of the manufacturer's processing software. The absolute calibration is based on the manufacturer-provided conversion from ring detector counts to physical units using radiant sensitivity of ring detectors $[59,60]$. Detection of scattered light within the angular range $14-155^{\circ}$ using the Roving Eyeball sensor employs measurements made with two linear polarization states of the incident beam and the corresponding two linear polarization states of the scattered light. The four measurement configurations allow for the determination of relative values of $p_{11}(\psi), p_{12}(\psi)$, and $p_{22}(\psi)$. The calibrated $\beta(\psi)$ values within the Roving Eyeball angular range are obtained by scaling the $p_{11}(\psi)$ data from the Roving Eyeball sensor. Specifically, the scattering measurements from the first angles of the Roving Eyeball sensor are forced to match the calibrated $\beta(\psi)$ values from the overlapping last ring detectors. The $\operatorname{DoLP}(\psi)$ values are obtained from Equation (2) using $p_{11}(\psi)$ and $p_{12}(\psi)$, and are also included in the standard output of the manufacturer's processing code.

We also used a DAWN-EOS multi-angle light scattering instrument which provided independent measurements of $\beta(\psi)$ and $\operatorname{DoLP}(\psi)$ of polystyrene beads suspended in water. These measurements were made with a sample placed in a $20 \mathrm{~mL}$ cylindrical glass vial. The DAWN-EOS instrument used in this study has been previously characterized and calibrated for such measurement configuration [61]. This instrument uses a diode-pumped frequency-doubled Nd-YAG laser at light wavelength $532 \mathrm{~nm}$ with a Gaussian beam profile of $62 \mu \mathrm{m}$ in diameter. The interrogated sample volume is on the order of $10 \mathrm{~nL}$. The incident beam can be linearly polarized both parallel and perpendicular to the scattering plane. The intensity of scattered light is measured simultaneously with eighteen photodiode detectors and no polarization analyzers, enabling measurements within a range of scattering angles from $22.5^{\circ}$ 
to $147^{\circ}$. To encompass the large dynamic range of scattered intensity, three selectable gain settings are available for each detector (gain factors of 1, 21, or 101).

As the DAWN-EOS detectors have no polarization analyzers, they only measure the first parameter of Stokes vector of the scattered light, $I_{s}(\psi)$. Here we define $I_{s \|}(\psi)$ for the parallel polarization of the incident beam and $I_{S \perp}(\psi)$ for the perpendicular polarization of the incident beam. The matrix elements $p_{11}(\psi)$ and $p_{12}(\psi)$ can be obtained (to within a constant factor) from DAWN-EOS measurements as

$$
\begin{aligned}
& p_{11}(\psi)=\frac{I_{s \|}(\psi)+I_{s \perp}(\psi)}{2} \\
& p_{12}(\psi)=\frac{I_{s \|}(\psi)-I_{s \perp}(\psi)}{2},
\end{aligned}
$$

which allows for determination of $\operatorname{DoLP}(\psi)$ from Equation (2). The calibration procedure described in Babin et al. [61] allows for determination of $\beta(\psi)$ in absolute units. Importantly, the calibration procedure of DAWN-EOS is fundamentally different from the calibration procedure of LISST-VSF. The manufacturer's calibration of LISST-VSF is based on a nominal radiant sensitivity of ring detectors (amperes of photoelectric current per watt of optical power) traceable to the National Institute of Standards and Technology [59,60]. In contrast, the calibration of DAWN-EOS is based on measurements of light scattered at $90^{\circ}$ by pure toluene with the incident beam having a linear perpendicular polarization [61]. This calibration relies on the known magnitude of molecular scattering by toluene. The two different methods employed in calibration of LISST-VSF and DAWN-EOS allow for comparisons of independent estimates of $\beta(\psi)$ obtained by these instruments. We also recall that the $\operatorname{DoLP}(\psi)$ estimates obtained with the two instruments within the common range of scattering angles are based on different polarization measurement configurations used by these instruments.

A Lambda 18 spectrophotometer was used to collect independent measurements of the spectral beam attenuation coefficient, $c(\lambda)$, of polystyrene beads suspended in water. These measurements were made for comparisons with the beam attenuation data obtained with LISST-VSF, and also to aid in the preparation of samples with appropriate concentrations of polystyrene beads to ensure that measurements with LISST-VSF and DAWN-EOS were made within the single scattering regime. The spectrophotometric measurements were made in the spectral range from $290 \mathrm{~nm}$ to $860 \mathrm{~nm}$ with $1 \mathrm{~nm}$ interval, but only data at $532 \mathrm{~nm}$ are used in this study. The general applicability of laboratory spectrophotometers with proper modifications to enable measurements of beam attenuation of particle suspensions, including colloidal samples, has long been recognized [62,63]. In our study, a sample of particle suspension was measured in a 1-cm quartz cuvette placed at a significant distance from the detector ( $\sim 25 \mathrm{~cm}$ from the entrance of the integrating sphere), and field stops were aligned within the light path to reduce the size of the beam and acceptance angle of the detector to less than $1^{\circ}$. This measurement geometry has been used in our previous studies of spectral beam attenuation by various particle assemblages $[64,65]$.

\subsubsection{Experimental Procedure}

Baseline measurements of $0.2 \mu \mathrm{m}$ filtered water were collected with all three instruments used in the experiments; LISST-VSF, DAWN-EOS, and Lambda 18 spectrophotometer. These baseline measurements were subtracted from subsequent measurements taken on particle suspensions to determine the optical properties associated with suspended particles only, i.e., the particulate volume scattering function, $\beta_{p}(\psi)$, the particulate degree of linear polarization, $\operatorname{DoLP}_{p}(\psi)$, and the particulate beam attenuation coefficient, $c_{p}$.

Original manufacturer's stock samples of standard polystyrene beads (100, 200, 400, 500, 700, and $2000 \mathrm{~nm}$ in diameter) were used to generate master samples using $0.2 \mu \mathrm{m}$ filtered, deionized, and degassed water as a medium (with the exception of $2000 \mathrm{~nm}$ beads which used $0.2 \mu \mathrm{m}$ filtered seawater). In the process of preparation of master samples, the particle concentration was optimized to ensure that spectrophotometric measurements of beam attenuation coefficient can be performed either 
directly or with small dilution factor $(\sim 3)$ on these samples over 1-cm path length with sufficiently high signal but negligible multiple scattering effects. The $c_{p}$ values for master samples ranged from about $18 \mathrm{~m}^{-1}$ to $58 \mathrm{~m}^{-1}$ (Table 1 ).

The master sample was diluted for measurements with the LISST-VSF to avoid oversaturation of PMT detectors and multiple scattering over the longer path length $(15 \mathrm{~cm})$. For baseline measurements, the LISST-VSF sample chamber was filled with $1900 \mathrm{~mL}$ of $0.2 \mu \mathrm{m}$ filtered water. The final samples of particle suspensions were created by addition of 20 to $100 \mathrm{~mL}$ of master sample to the LISST-VSF chamber. For most beads examined in our experiments, more than one particle suspension differing in terms of particle concentration was measured with LISST-VSF (Table 1). The different particle concentrations were achieved by different dilution of master sample within LISST-VSF chamber. Owing to different dilution factors ranging from 20 to 96 (labeled as DF1, DF2, and DF3 in Table 1), the $c_{p}$ values of LISST-VSF samples ranged from about $0.5 \mathrm{~m}^{-1}$ to $1.8 \mathrm{~m}^{-1}$. For a single bead size, concentration, and PMT gain, a series of LISST-VSF measurements was composed of 200 measurements taken in rapid succession (recall that a measurement refers to two scans, each with a different polarization of incident beam). This measurement series was divided into five sets of 20 measurements and one set of 100 measurements to enable manual gentle mixing of sample before each set of measurements. In addition, for the $2000 \mathrm{~nm}$ bead suspensions a magnetic stir bar which operated on low speed and changed direction of rotation every $30 \mathrm{~s}$ was used to prevent particle settling during the measurement.

Several LISST-VSF baseline measurements of $0.2 \mu \mathrm{m}$ filtered water were collected for each experiment, i.e., for each examined bead size. However, for reasons of consistency and out of the desire to use an optimal baseline representative of the least contaminated $0.2 \mu \mathrm{m}$ filtered water, a single baseline was used for processing of all experimental data collected for various bead sizes and concentrations except for $2000 \mathrm{~nm}$ sized beads which used $0.2 \mu \mathrm{m}$ filtered seawater. This baseline was determined on the basis of finding a measurement which exhibited minimal scattering signal detected by Roving Eyeball and ring detectors and maximum directly transmitted light detected by the laser transmission sensor. We note, however, that for each PMT gain setting of the Roving Eyeball sensor a separate baseline was determined.

Measurements using the DAWN-EOS instrument were collected for 100, 200, 400, and $700 \mathrm{~nm}$ beads. Dilution factors of master samples for DAWN-EOS measurements were between 300 and 3000 , depending on bead size. The gain settings for each detector were adjusted to the highest setting that would avoid saturation of signal with incident perpendicular polarization of light. For 400 and $700 \mathrm{~nm}$ bead suspensions, two different dilutions were measured. For each polarization state (i.e., perpendicular and parallel) of incident light, we acquired 1440 measurements with a sampling frequency of $8 \mathrm{~Hz}$ over $3 \mathrm{~min}$. For a given sample, this data acquisition protocol was repeated three times. Each of these three replications was made with a different randomly-chosen orientation of sample cylindrical vial within the instrument. The sample was gently mixed between these replicate measurements. The baseline measurements of $0.2 \mu \mathrm{m}$ filtered water were acquired using the same protocol.

As mentioned above, the optical measurements were made on sufficiently-diluted samples to ensure negligible effects of multiple scattering over a pathlength used by a given instrument. A criterion for a single scattering regime is generally defined in terms of small optical thickness of the sample, $\tau<<1$, where $\tau$ is a product of the beam attenuation coefficient, $c$, and pathlength, $r[51,66]$. Also, a simple practical test for ensuring that multiple scattering effects are negligible is to verify a direct proportionality between the measured optical signal and the concentration of particles in suspension by conducting a series of measurements on the same sample with different dilutions [51]. Our measurements on bead samples with different dilutions showed an excellent 1:1 relationship between the LISST-VSF measurement and the bead concentration over the range of beam attenuation coefficient up to at least $2 \mathrm{~m}^{-1}$. The single scattering regime can also be determined by the condition $\tau(1-g)<<1$, where $g$ is the average cosine of the scattering angle of the volume scattering function $[23,66]$. For the $100 \mathrm{~nm}$ polystyrene beads, the $g$ value is 0.115 , which yields the 
most restrictive condition in our study, $\tau<<1.13$. For all bead samples measured with LISST-VSF, including all bead sizes and sample dilutions, $\tau$ was always less than about 0.3 . This condition was also satisfied for samples measured with a spectrophotometer. For the measurements with DAWN-EOS, the $\tau$ values were even smaller. For the natural seawater samples examined in our study (which is described below in Section 2.2), the $g$ values (for the total volume scattering function including the contribution by pure seawater) were about 0.9 or somewhat higher, which yields less restrictive criterion $\tau<<10$. Our measurements of natural samples clearly satisfied this single scattering condition, as the highest value of $c$ for the natural samples measured with LISST-VSF was about $2.6 \mathrm{~m}^{-1}$, so $\tau$ was always less than about 0.45 , given that the maximum pathlength for LISST-VSF is $17.5 \mathrm{~cm}$ for the scattering angle of $150^{\circ}$.

\subsubsection{Data Processing}

Processing of LISST-VSF data was done with a standard processing code provided by manufacturer (version of 2013) to determine $\beta_{p}(\psi), \operatorname{DoLP} P_{p}(\psi)$, and $c_{p}$, denoted hereafter as $\beta_{p}^{\operatorname{LISST*}}(\psi)$, $\operatorname{DoLP}_{p}^{L I S S T *}(\psi)$, and $c_{p}^{L I S S T *}$ respectively (the asterisk indicates that the variable is derived from the standard processing code without additional corrections developed in this study). Some details specific to the processing and quality control of our experimental data are provided below.

As a first step in data processing, the baseline values in raw counts were subtracted from each LISST-VSF measurement of raw counts acquired on samples of bead suspensions. To account for light attenuation along the path between the scattering volume and the detector, an attenuation correction factor was calculated using the average $c_{p}^{L I S S T *}$ from the series of measurements and the length of the path for each scattering angle. Further, to account for the difference in sensitivity of the two Roving Eyeball PMT detectors, a factor $\alpha$ is used to adjust the measured counts of one PMT detector relative to the other [67]. The value of $\alpha=0.9335$ was determined by averaging all median values of $\alpha$ derived from each series of measurements for each bead size, particle concentration, and PMT gain. The $\alpha$ parameter was observed to be nearly constant over the period of experiments ( 18 months, the coefficient of variation $<5 \%$ ). For each series of measurements a specific scaling factor was determined to convert $p_{11}(\psi)$ in PMT counts to $\beta_{p}^{L I S S T *}(\psi)$ in absolute units $\left[\mathrm{m}^{-1} \mathrm{sr}^{-1}\right]$ for scattering angles $14-155^{\circ}$ measured by the Roving Eyeball sensor. First, for each measurement from a given series of measurements, a scaling factor was determined by matching the PMT counts measured with Roving Eyeball sensor between $15^{\circ}$ and $16^{\circ}$ with $\beta_{p}^{L I S S T *}(\psi)$ in absolute units obtained from measurements with the last two ring detectors at $13.01^{\circ}$ and $15.17^{\circ}$. Then, using these determinations, the average scaling factor for a given series of measurements was calculated and used for further data processing. Note that this scaling was not needed for the determination of $\operatorname{DoLP}_{p}^{L I S S T *}(\psi)$ for the Roving Eyeball angular range, which is calculated from $p_{11}(\psi)$ and $p_{12}(\psi)$ determined in PMT counts following Equation (2).

Quality control of data was performed by removing the first set of 20 measurements (the remaining four sets with 20 measurements each were retained) and the first 20 measurements from the set of 100 measurements. We observed that this was necessary to ensure reasonable stability in the measured scattering signal. The mean and standard deviation values for each angle based on all of the 160 remaining measurements in the series were determined, and the outlying single measurements within the series were identified and rejected from subsequent analysis. Typically, 120 to 130 measurements from a given series of 200 measurements passed the quality criteria.

Example data of uncorrected $\beta_{p}^{L I S S T *}(\psi)$ for $200 \mathrm{~nm}$ and $2000 \mathrm{~nm}$ bead suspensions are shown in Figure 1. The series of measurements that remained after quality control and the median values of $\beta_{p}^{\text {LISST* }}(\psi)$ derived from the series of measurements are shown. We also note that the median values were very close to mean values for our data $(<1 \%$ difference for most scattering angles). The results for $2000 \mathrm{~nm}$ beads show a distinct pattern with several scattering maxima and minima due to constructive and destructive interference of the scattered light from a nearly monodisperse population of beads that are large relative to the wavelength of light. The $200 \mathrm{~nm}$ beads are smaller 
than the wavelength of light leading to a more featureless shape of $\beta_{p}^{L I S S T *}(\psi)$. The variability between the individual measurements is largest at very small scattering angles, i.e., approximately $<4^{\circ}$, where the scattering signal for submicron particles is low relative to our baseline measurements. Apart from small scattering angles, the coefficient of variation $(\mathrm{CV})$ for each scattering angle calculated from a series of measurements on $200 \mathrm{~nm}$ beads is generally very small, ranging from $\sim 3 \%$ to $<1 \%$, with the smallest values at angles greater than $64^{\circ}$ where full laser power is used. The measurements of $2000 \mathrm{~nm}$ beads exhibit somewhat higher $C V$, i.e., between about $3 \%$ and $6 \%$. The higher values of $C V$ are observed mostly near the angles where minima of $\beta_{p}^{L I S S T *}(\psi)$ occur.
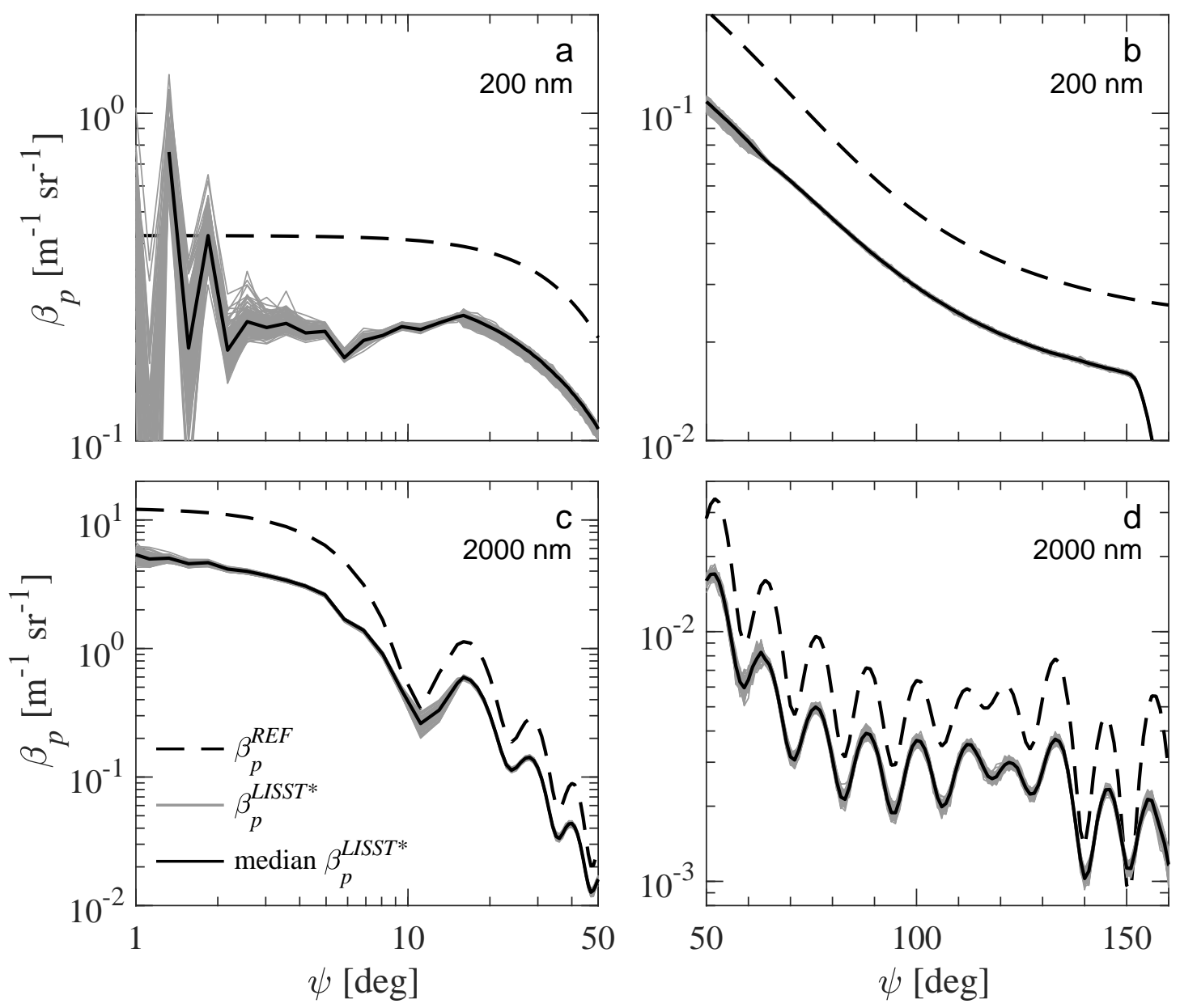

Figure 1. Measurements of the particulate volume scattering function, $\beta_{p}(\psi)$, at light wavelength of $532 \mathrm{~nm}$ for $200 \mathrm{~nm}(\mathbf{a}, \mathbf{b})$ and $2000 \mathrm{~nm}(\mathbf{c}, \mathbf{d})$ diameter polystyrene beads suspended in water. The left panels depict the angular range of $1-50^{\circ}$ with logarithmic scaling, and the right panels depict the range $50-160^{\circ}$ with linear scaling. The expected reference value, $\beta_{p}^{R E F}(\psi)$, obtained from Mie scattering calculations is indicated as a dashed line. Quality-controlled but uncorrected measurements obtained with the LISST-VSF (gray lines, number of measurements $N=128$ ) and the median value (solid black line) are shown.

The DAWN-EOS measurements for four bead sizes were also used to calculate $\beta_{p}(\psi)$ and $\operatorname{DoLP}_{p}(\psi)$, denoted as $\beta_{p}^{\mathrm{DAWN}}(\psi)$ and $\operatorname{DoLP} P_{p}^{\mathrm{DAWN}}(\psi)$. First, for each time series of 1440 measurements with DAWN-EOS, the highest $2 \%$ of data was rejected, as these data are assumed to result from sample contamination with rare, larger particles. Each set of measurements then consists of 1411 measurements of both $I_{s \|}(\psi)$ and $I_{s \perp}(\psi)$ for a specific orientation of sample vial. These measurements were averaged to represent that orientation. Such results were then averaged for three vial orientations. This protocol was applied to both the sample and baseline measurements, with the exception that 
baseline values were calculated by averaging the lowest $5 \%$ of data. The final $I_{s \|}(\psi)$ and $I_{S \perp}(\psi)$ for the beads were calculated by subtracting the average baseline from the average sample data. These particulate $I_{s \|}(\psi)$ and $I_{s \perp}(\psi)$ were then used to determine $p_{11}(\psi)$ and $p_{12}(\psi)$ according to Equations (3) and (4), from which $\beta_{p}^{\mathrm{DAWN}}(\psi)[61]$ and $\operatorname{DoL} P_{p}^{\mathrm{DAWN}}(\psi)$ (Equation (2)) were determined. Note that two dilutions of the master suspension for 400 and $700 \mathrm{~nm}$ beads were measured with DAWN-EOS and the average of the two was used to represent these bead sizes. As a final step, the determined $\beta_{p}^{\mathrm{DAWN}}(\psi)$ values were rescaled using relevant dilution factors to obtain final results representing the particle concentration in LISST-VSF samples and enable direct comparisons with LISST-VSF measurements. Note that such rescaling is not necessary for $\operatorname{DoLP}_{p}^{\mathrm{DAWN}}(\psi)$.

With regard to processing of data acquired with a Lambda 18 spectrophotometer, the spectral data of measured optical density $\operatorname{OD}(\lambda)$ (i.e., measurements made in the absorbance mode of the spectrophotometer) were converted (after subtraction of baseline measurement) into the particulate beam attenuation coefficient $\left[\mathrm{m}^{-1}\right]$ using the relationship $c_{p}(\lambda)=\ln (10) \mathrm{OD}(\lambda) / 0.01$, where $\ln$ is the natural logarithm and 0.01 is the path length in meters. The final particulate beam attenuation coefficient obtained from spectrophotometric measurements is denoted as $c_{p}^{S P E C}$. The estimates of $c_{p}^{L I S S T *}$ from LISST-VSF measurements were calculated with the standard manufacturer's processing code. Because each LISST-VSF measurement consists of two linear polarization states of the incident beam, the average of these two is used as the final estimate of $c_{p}^{L I S S T *}$. As a final step, the determined $c_{p}^{S P E C}$ values were multiplied by relevant dilution factors to obtain final results representing particle concentration in LISST-VSF samples and enable direct comparisons with LISST-VSF measurements.

\subsubsection{Determination of Correction Functions}

In addition to $\beta_{p}^{\operatorname{LISST} *}(\psi)$, Figure 1 shows results for the $200 \mathrm{~nm}$ and $2000 \mathrm{~nm}$ polystyrene beads based on Mie scattering calculations (more details about these calculations are provided below). These results are significantly higher (nearly a factor of 2) than the measured values of $\beta_{p}^{L I S S T *}(\psi)$. We assume that the Mie scattering calculations for samples of spherical polystyrene beads are sufficiently accurate to provide reference values for such samples.

In order to correct for the mismatch between the measured and reference values, a calibration correction function $C F(\psi)$ is defined as

$$
C F(\psi)=\frac{\beta_{p}^{R E F}(\psi)}{\beta_{p}^{L I S S T *}(\psi)},
$$

where $\beta_{p}^{R E F}(\psi)$ is a reference volume scattering function determined according to

$$
\beta_{p}^{R E F}(\psi)=\widetilde{\beta}_{p}^{M i e}(\psi) b_{p}^{R E F},
$$

where $\widetilde{\beta}_{p}^{M i e}(\psi)$ is the scattering phase function $\left[\mathrm{sr}^{-1}\right]$ obtained from Mie scattering computations and $b_{p}^{R E F}$ is the reference particulate scattering coefficient $\left[\mathrm{m}^{-1}\right]$. Note that all quantities in Equations (5) and (6) are for the LISST-VSF light wavelength of $532 \mathrm{~nm}$.

For each examined suspension of standard polystyrene beads, $\widetilde{\beta}_{p}^{\text {Mie }}(\psi)$ was determined from Mie scattering computations for homogeneous spherical particles. We used the Mie scattering code for homogeneous spheres of Bohren and Huffman [20], which included our modifications to account for polydispersity of the sample, i.e., to use particle size distribution as input to the code rather than just a single particle diameter as in the original code. The computations were performed assuming a relative particle size distribution (PSD) of Gaussian shape, with 300 evenly spaced size bins about the nominal mean diameter \pm 3 standard deviations, as provided by the manufacturer for each bead size (Table 1 ). The use of such PSDs allows us to account for the realistic, small degree of polydispersity of each sample. The Mie computations also require input of the refractive index of particles. Based on the study of Ma et al. [68] we assumed that the complex refractive index of polystyrene relative to water at 
$532 \mathrm{~nm}$ is $m=1.193+0.0003 i$, where the first component is the real part and the second component is the imaginary part of refractive index. Note that the imaginary part is very small because polystyrene is a weakly absorbing material in the examined spectral region.

Equation (6) also requires $b_{p}^{R E F}$, which was determined from the combination of beam attenuation measurements and Mie scattering calculations as

$$
b_{p}^{R E F}=c_{p}^{L I S S T *} \frac{Q_{b}^{M i e}}{Q_{c}^{M i e}}
$$

where $Q_{b}^{\text {Mie }}$ and $Q_{c}^{\text {Mie }}$ are the single-particle scattering and attenuation efficiency factors, respectively, obtained from Mie computations. Because the populations of examined beads exhibit a slight degree of polydispersity, the calculated $Q_{b}^{\mathrm{Mi} i}$ and $Q_{c}^{\mathrm{Mie}}$ represent the average values of efficiency factors for a given particle population [69]. Given very weak light absorption of polystyrene beads at $532 \mathrm{~nm}$, the ratio $\frac{Q_{b}^{\text {Mie }}}{Q_{c}^{\text {Mie }}}$ was found to be $>95 \%$. We also note that in addition to $c_{p}^{\text {LISST } *}$, we have another potential measurement of beam attenuation coefficient from the spectrophotometer $\left(c_{p}^{S P E C}\right)$. Figure 2 shows that the measurements of $c_{p}^{L I S S T *}$ and $c_{p}^{S P E C}$ are consistent, and generally agree very well.

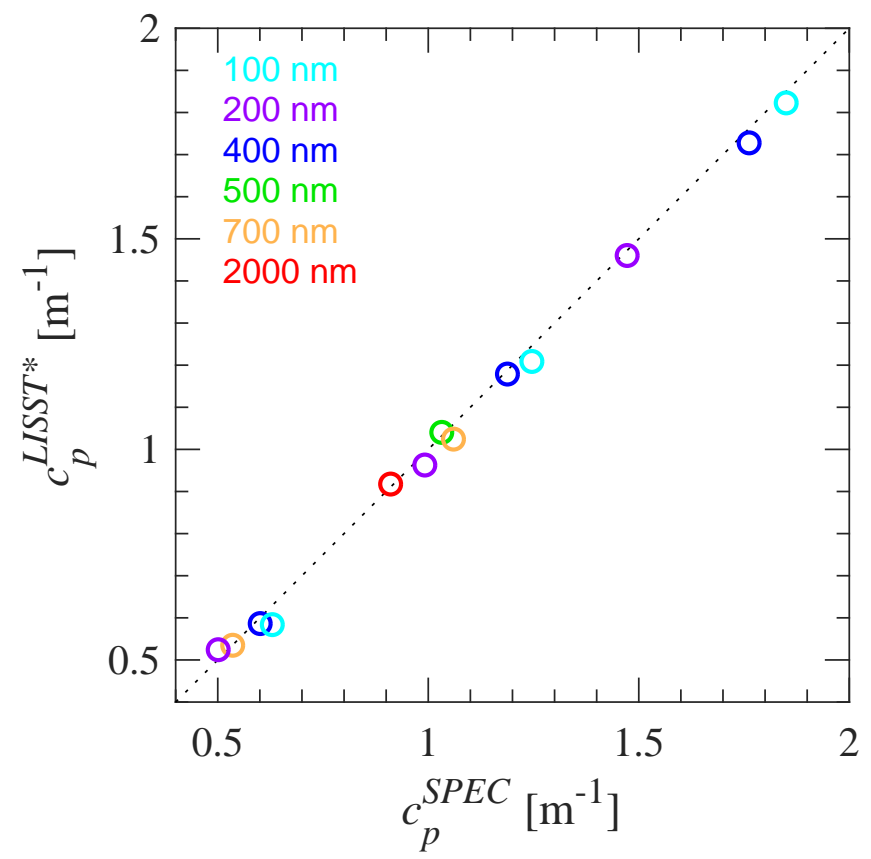

Figure 2. Comparison of measurements of the particulate beam attenuation coefficient, $c_{p}$, at $532 \mathrm{~nm}$ obtained with a spectrophotometer with measurements from the LISST-VSF. The comparison is depicted for suspensions of polystyrene beads of six different diameters as indicated in the legend, and the 1:1 line is plotted for reference (dotted black line). Appropriate dilution factors have been applied to account for the different particle concentrations used in measurements with each instrument. The presented values correspond to samples measured with the LISST-VSF.

By combining Equations (6) and (7), $\beta_{p}^{R E F}(\psi)$ can be determined for each LISST-VSF measurement as

$$
\beta_{p}^{R E F}(\psi)=\widetilde{\beta}_{p}^{M i e}(\psi) c_{p}^{L I S S T *} \frac{Q_{b}^{M i e}}{Q_{c}^{M i e}} .
$$

Note that the estimates of $\beta_{p}^{R E F}(\psi)$ can vary between individual measurements because of variations in $c_{p}^{L I S S T *}$. Finally, by combining Equations (5) and (8), $C F(\psi)$ can be determined for each LISST-VSF measurement as 


$$
C F(\psi)=\frac{\widetilde{\beta}_{p}^{M i e}(\psi) c_{p}^{L I S S T *} \frac{Q_{b}^{\text {Mie }}}{Q_{c}^{\text {Mie }}}}{\beta_{p}^{L I S S T *}(\psi)}
$$

The application of this protocol to every individual measurement of $\beta_{p}^{L I S S T *}(\psi)$ helps to better capture the variability between individual measurements during a given series of LISST-VSF measurements on a given sample, for example due to imperfect mixing in the $2 \mathrm{~L}$ sample chamber or potential electronic fluctuations in the instrument.

The smaller-sized particle standards ( $<500 \mathrm{~nm}$ in diameter) appear as the best candidates for determination of $C F(\psi)$ because they produce a relatively featureless pattern of angular scattering (see the results for $200 \mathrm{~nm}$ beads in Figure 1). The measurements with larger beads ( $500 \mathrm{~nm}$ to $2000 \mathrm{~nm}$ ) were not used in these determinations because the angular scattering pattern includes multiple maxima and minima (see the results for $2000 \mathrm{~nm}$ beads in Figure 1), which render the comparison of $\beta_{p}^{L I S S T *}(\psi)$ and $\beta_{p}^{R E F}(\psi)$ particularly sensitive to even small uncertainties in measurements or theoretical calculations. The results obtained with beads of 100, 200, and $400 \mathrm{~nm}$ in diameter were considered in the determinations of final correction function $C F_{f}(\psi)$ within four angular ranges, as described below:

$$
\begin{gathered}
C F_{f}(\psi)=\left(C F_{100}(\psi)+C F_{200}(\psi)+C F_{400}(\psi)\right) / 3 \text { for } \psi=0.09-60^{\circ} \\
C F_{f}(\psi)=C F_{200}(\psi) \text { for } \psi=61-128^{\circ} \\
C F_{f}(\psi)=\left(C F_{200}(\psi)+C F_{400}(\psi)\right) / 2 \text { for } \psi=129-150^{\circ} \\
C F_{f}(\psi)=\left(C F_{100}(\psi)+C F_{200}(\psi)+C F_{400}(\psi)\right) / 3 \text { for } \psi=151-155^{\circ} .
\end{gathered}
$$

The $C F_{200}(\psi)$ data obtained with $200 \mathrm{~nm}$ beads provide the main contribution to the determination of $C F_{f}(\psi)$. The $C F_{100}(\psi)$ data obtained with $100 \mathrm{~nm}$ beads are used partially because of increased uncertainty in the PSD of these beads ( $C V$ of nominal mean diameter is $7.8 \%$, see Table 1 ). The $C F_{400}(\psi)$ data obtained with $400 \mathrm{~nm}$ beads are also used partially and cover the backscattering angles, where these particular data are useful for correction of an apparent artifact near $130-140^{\circ}$, which is rather minor but has been consistently observed with our LISST-VSF instrument for various natural particle assemblages. The calculations of $C F_{100}(\psi), C F_{200}(\psi)$, and $C F_{400}(\psi)$ were made using data for particle concentrations and PMT gains which ensured sufficient signal for the ring detectors while avoiding PMT saturation of the Roving Eyeball sensor (see dilution factors in italic font in Table 1). For a given bead standard, the final values of correction function at different angles were determined as the median values of all the relevant determinations.

The final $C F_{f}(\psi)$ was smoothed in the angular range $2.56-155^{\circ}$ with a 3-point and then a 5-point moving average. In addition, $C F_{f}(\psi)$ within the near-forward angular range $0.09-4.96^{\circ}$ was set to a constant value of $C F_{f}\left(\psi_{32}\right)$, where $\psi_{32}=15.17^{\circ}$ corresponds to the last ring detector. The rationale for this assumption is that the scattering signal produced by the examined beads for the first 25 rings $\left(\psi=0.09-4.96^{\circ}\right)$ is comparable to the baseline, while there is good signal relative to the baseline for the last ring detector.

The final correction simply involves the multiplication of uncorrected $\beta_{p}^{L I S S T *}(\psi)$ by the correction function $C F_{f}(\psi)$,

$$
\beta_{p}^{\operatorname{LISST}}(\psi)=\beta_{p}^{\operatorname{LISST*}}(\psi) C F_{f}(\psi),
$$

where $\beta_{p}^{L I S S T}(\psi)$ is the corrected LISST-VSF measurement of volume scattering function (note that the superscript * is removed from this symbol).

We also determined a correction function for $\operatorname{DoLP}_{p}^{L I S S T *}(\psi)$,

$$
B F(\psi)=\operatorname{DoLP}_{p}^{L I S S T *}(\psi)-\operatorname{DoLP}_{p}^{R E F}(\psi)
$$


where $B F(\psi)$ quantifies a correction for potential bias in $\operatorname{DoLP}_{p}^{L I S S T *}(\psi)$ obtained from the standard processing code applied to LISST-VSF measurements and $\operatorname{DoLP} P_{p}^{R E F}(\psi)$ is a reference degree of linear polarization determined from Mie scattering calculations of the two scattering matrix elements, $p_{11}^{\text {Mie }}(\psi)$ and $p_{12}^{\text {Mie }}(\psi)$, for a given sample of standard beads. The results for $B F_{100}(\psi), B F_{200}(\psi), B F_{400}(\psi)$, and the final correction function $B F_{f}(\psi)$ were obtained using a procedure similar to that for $C F_{100}(\psi), C F_{200}(\psi), C F_{400}(\psi)$, and $C F_{f}(\psi)$. The correction of $\operatorname{DoLP}_{p}^{L I S S T *}(\psi)$ simply requires a subtraction of $B F_{f}(\psi)$,

$$
\operatorname{DoLP}_{p}^{L I S S T}(\psi)=\operatorname{DoLP}_{p}^{L I S S T *}(\psi)-B F_{f}(\psi),
$$

where $\operatorname{DoLP} P_{p}^{L I S S T}(\psi)$ is the corrected degree of linear polarization within the range of scattering angles from $16^{\circ}$ to $150^{\circ}$. Because the $\operatorname{DoLP} P_{p}^{L I S S T *}(\psi)$ data output from standard processing of LISST-VSF measurements begins at $\psi=16^{\circ}$, no correction for the forward scattering angles of the ring detectors $\left(\psi<16^{\circ}\right)$ was determined.

\subsection{Measurements and Analysis of Natural Seawater Samples}

Optical measurements with the LISST-VSF and ancillary analyses of natural particle assemblages were performed on seawater samples collected between summer 2016 and spring 2017 in contrasting marine environments, namely, in open ocean waters off the coast of Southern California, nearshore ocean waters at the pier of the Scripps Institution of Oceanography (SIO Pier) in La Jolla, and the tidal estuary of the San Diego River. Overall 17 samples representing a broad range of natural particle assemblages were analyzed. Most samples (number of samples $N=11$ ) were collected at the SIO Pier. These samples were collected during typical dry weather conditions, phytoplankton bloom events, and after heavy rain. The tidal estuary samples $(N=3)$ include three tidal states between low and high tide. The offshore samples $(N=3)$ were collected in the Santa Barbara Channel, about $8 \mathrm{~km}$ off San Diego Bay, and about $2 \mathrm{~km}$ off SIO Pier. Seawater samples were collected just beneath the sea surface using either Niskin bottles or a bucket, except for one offshore sample (off San Diego Bay) that was collected at the subsurface chlorophyll- $a$ maximum at a depth of $18 \mathrm{~m}$. All samples were analyzed in the laboratory within $24 \mathrm{~h}$ of sampling.

To characterize the concentration and composition of particulate matter for each sample, we determined the dry mass concentration of total suspended particulate matter, SPM $\left[\mathrm{g} \mathrm{m}^{-3}\right]$, mass concentration of particulate organic carbon, POC $\left[\mathrm{mg} \mathrm{m}^{-3}\right]$, and mass concentration of the pigment chlorophyll- $a$, Chla $\left[\mathrm{mg} \mathrm{m}^{-3}\right]$. For these determinations, the particles were collected on glass-fiber filters (GF/F Whatman) by filtration of appropriate volumes of seawater (150-2100 mL depending on the sample). SPM was determined following a gravimetric method using pre-washed and pre-weighted filters $[7,70]$. The determinations of POC were made on precombusted filters with a standard CHN analysis involving high temperature combustion of sample filters [7,71,72]. Chla was determined spectrophotometrically using a Lambda 18 spectrophotometer and placing $1-\mathrm{cm}$ cuvettes containing acetone extracts of the samples inside the integrating sphere. The measured absorbance values at 630,647, 665, and $691 \mathrm{~nm}$ (after subtraction of acetone baseline values) were used in the calculation of Chla [73]. For each seawater sample, replicate determinations of SPM and POC were made on separate sample filters. The final SPM and POC are average values of replicate determinations. The replicates for SPM and POC agreed generally to within $15 \%$ and $10 \%$, respectively. No replicates were taken for Chla. In addition to information about particle concentration, SPM, POC, and Chla provide useful proxies of bulk composition of particulate matter. The organic and inorganic fractions of SPM can be characterized using the ratio POC/SPM, and the contribution of phytoplankton to SPM using Chla/SPM [65]. These ratios are expressed on a [g/g] basis.

The measurements of particle size distribution (PSD) were made with a Coulter Multisizer 3 (Beckman Coulter, Brea, CA, USA) equipped with a $100 \mu \mathrm{m}$ aperture, which allows particle counting and sizing in the range of volume-equivalent spherical diameter from $2 \mu \mathrm{m}$ to $60 \mu \mathrm{m}$. Within this size range we used 300 log-spaced size bins to provide high resolution PSDs. For each experiment, $0.2 \mu \mathrm{m}$ filtered seawater was used as a blank that was subtracted from sample measurements. 
Approximately 10 to 15 replicate measurements of $2 \mathrm{~mL}$ subsamples of each seawater sample were collected. After removing outliers, the remaining measurements were summed and divided by the total analyzed volume to produce an average density function of PSD in particle number per unit volume per width of size bin. For each sample the power function fit with a slope parameter, $\zeta$, was determined using these PSD data over the size range 2-50 $\mu \mathrm{m}$. In these determinations, the linear regression analysis was applied to log-transformed data, and the last size bins with very low particle counts were ignored. Although the measured PSDs often showed significant deviations from the power function fits, we use the slope parameter $\zeta$ as a particle size metric, because this is the most common parameterization of size distribution of marine particles [23,74]. Additionally, assuming spherical particles, the particle volume distributions were determined from particle number distributions for each sample. From particle volume distributions, we calculated the percentile-based particle diameters such as the median diameter, $D_{V}^{50}$, and the $90^{\text {th }}$ percentile diameter, $D_{V}^{90}$. These parameters have been shown to provide potentially useful metrics in the analysis of relationships between the optical and particle size properties in seawater [65].

Measurements and processing of data collected with LISST-VSF for natural seawater samples were made following a protocol similar to that described above for standard polystyrene bead samples. For each experiment, baseline measurements were taken on $0.2 \mu \mathrm{m}$ filtered seawater obtained from a given seawater sample. However, a single baseline selected from the lowest measured baselines was used for data processing of all seawater samples to ensure a consistent baseline unaffected by possible variations associated with the imperfect purity of $0.2 \mu \mathrm{m}$ filtered seawater prepared during different experiments. To ensure scattering measurements were acquired in a single-scattering regime, samples with an average $c_{p}$ over $3.0 \mathrm{~m}^{-1}$ were diluted using $0.2 \mu \mathrm{m}$ filtered seawater. Dilution was necessary only for the two most turbid samples collected in the San Diego River Estuary. Between four and eight sets of 50 measurements were collected for each seawater sample with gentle hand mixing between the measurement sets, while a magnetic stir bar was on very low speed changing direction of rotation every $30 \mathrm{~s}$. All results from LISST-VSF measurements for natural seawater samples shown in this paper represent the $C F_{f}(\psi)$-corrected volume scattering function of particles, $\beta_{p}^{\operatorname{LISST}}(\psi)$, and $B F_{f}(\psi)$-corrected degree of linear polarization of particles, $\operatorname{DoLP}_{p}^{L I S S T}(\psi)$. For a given sample the final values of $\beta_{p}^{\operatorname{LSST}}(\psi)$ and $\operatorname{DoLP}_{p}^{L I S S T}(\psi)$ correspond to the median values of the series of measurements that passed the quality control criteria.

To determine the particulate scattering, $b_{p}^{L I S S T}$, and particulate backscattering, $b_{b p}^{L I S S T}$, coefficients, the corrected measured $\beta_{p}^{L I S S T}(\psi)$ was first extrapolated in the angular range 150-180. The extrapolated portion of $\beta_{p}^{L I S S T}(\psi)$ was obtained by fitting a specific function to the data of $\beta_{p}^{L I S S T}(\psi)$ in the angular range $90-150^{\circ}$. We used two methods for fitting and extrapolating $\beta_{p}^{L I S S T}(\psi)$. The first method is based on a non-linear least squares best fit of the analytical function proposed by Beardsley and Zaneveld [75]. The second method is based on a linear mixing model that finds a non-negative least squares best fit for combined contributions of four end members representing shapes of volume scattering functions associated with scattering by small and large particles, as described in Zhang et al. [76].

A backscattering factor, $\kappa$, was determined for the fitted volume scattering function as

$$
\kappa=\frac{b_{b p}^{f i t}}{b_{b p, 150}^{\text {fit }}}
$$

where $b_{b p}^{\text {fit }}$ is the particulate backscattering coefficient determined by the integration of the fitted function in the angular range $90-180^{\circ}$ and $b_{b p, 150}^{\text {fit }}$ is the coefficient determined by the integration of the fitted function in the range $90-150^{\circ}$. The final estimate of backscattering coefficient, $b_{b p}^{L I S T}$, was calculated as

$$
b_{b p}^{L I S S T}=\kappa b_{b p, 150}^{L I S S T}
$$


where $b_{b p, 150}^{L I S T}$ is obtained by the integration of $\beta_{p}^{L I S S T}(\psi)$ in the angular range $90-150^{\circ}$. The final estimate of scattering coefficient, $b_{p}^{L I S S T}$, was calculated as the sum of $b_{b p}^{L I S S T}$ and the forward scattering coefficient obtained from the integration of $\beta_{p}^{L I S S T}(\psi)$ in the angular range $0.09-90^{\circ}$.

The calculations of $b_{p}^{\text {LIST }}$ and $b_{b p}^{\text {LISST }}$ were made for each seawater sample using the two methods for fitting and extrapolation. The particulate backscattering ratio, $\widetilde{b}_{b p}^{\text {LISST }}=b_{b p}^{\text {LISST }} / b_{p}^{\text {LISST }}$ was also calculated. We note that the $\kappa$ values for all examined seawater samples were found to range between 1.125 and 1.138 and 1.118-1.120 for the Beardsley and Zaneveld [75] and Zhang et al. [76] methods, respectively. An example illustration of fitting and extrapolation methods for one sample collected during high tide at the San Diego River estuary is depicted in Figure 3. As seen, both the Beardsley and Zaneveld [75] and Zhang et al. [76] fitted functions are in good agreement with the measured data of $\beta_{p}^{L I S T}(\psi)$ in the angular range $90-150^{\circ}$. However, the extrapolated portion of the Beardsley and Zaneveld [75] function in the angular range $150-180^{\circ}$ has somewhat higher values compared with the Zhang et al. [76] function. Nevertheless, the estimates of $b_{b p}^{\text {LISST }}$ for this sample obtained from the two extrapolation methods differ only by $0.5 \%$. For all other seawater samples the difference was also small, not exceeding $1.5 \%$. The final results of $b_{p}^{\text {LISST }}$ and $b_{b p}^{\text {LIST }}$ for seawater samples presented in this study are based on the Zhang et al. [76] method.

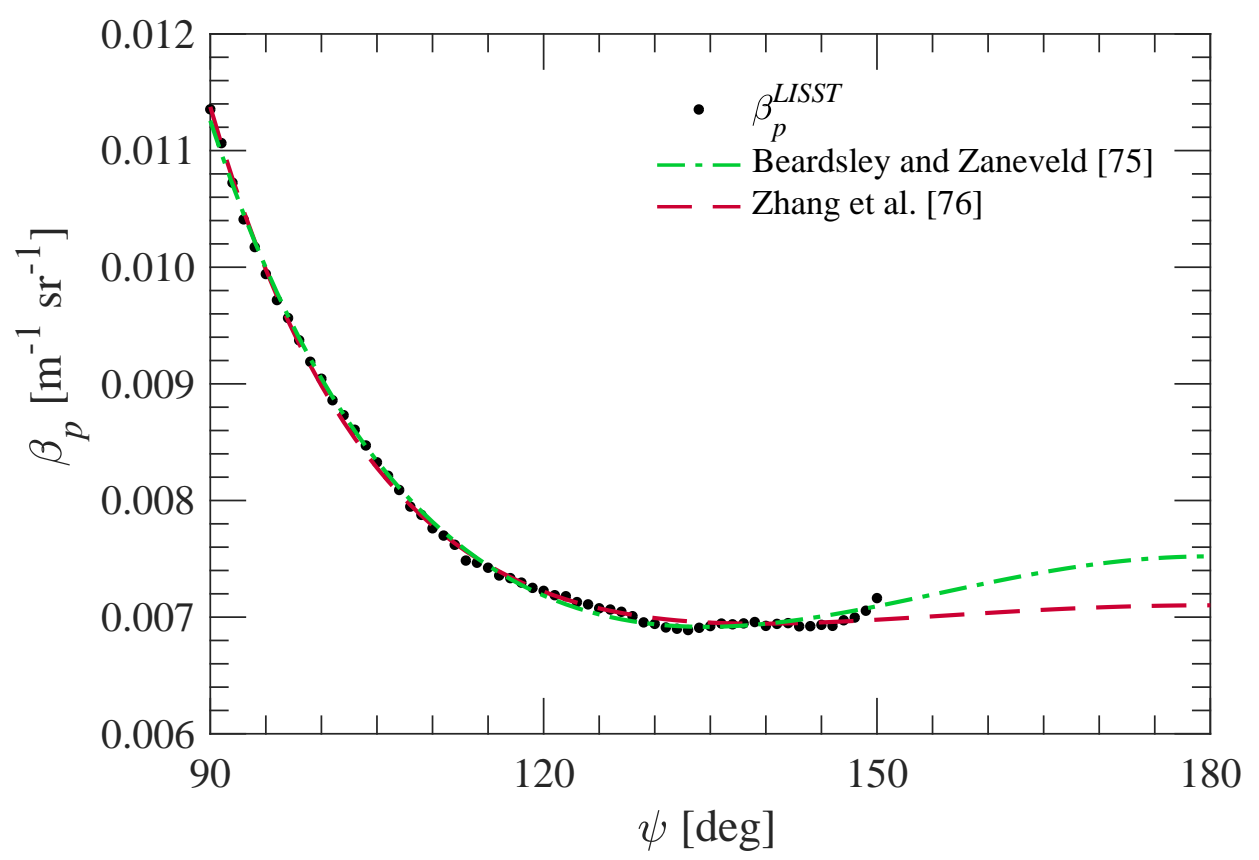

Figure 3. Measured values of the particulate volume scattering function $\beta_{p}(\psi)$ obtained with the LISST-VSF after correction (circles) for scattering angles $90-150^{\circ}$ and illustration of the results of two model relationships (Beardsley and Zaneveld [75], Zhang et al. [76]) fitted to the data. The illustrated example measurement was made on a natural sample collected from the San Diego River estuary.

\section{Results and Discussion}

\subsection{Correction Functions for LISST-VSF}

The results for $C F_{100}(\psi), C F_{200}(\psi), C F_{400}(\psi)$, and $C F_{f}(\psi)$ are plotted in Figure 4. The final correction function $C F_{f}(\psi)$ indicates that $\beta_{p}^{L I S S T *}(\psi)$ is lower than $\beta_{p}^{R E F}(\psi)$ by a factor of about 2, and also exhibits some angular variability. One consistent feature in the forward scattering region, which is independent of the bead size, is a sharp increase in $C F_{f}(\psi)$ with a peak at ring $26\left(\psi=5.84^{\circ}\right)$. We observed a similar but inverse feature consistently in natural seawater samples, which suggests that the behavior of the correction function at these angles is credible. Within the angular range of data from the Roving Eyeball sensor $\left(16-150^{\circ}\right)$, the $C F_{f}(\psi)$ values remain generally in the range between 
1.7 and 1.9. For angles larger than $150^{\circ}$, we did not obtain consistent results of the correction function for different bead sizes (not shown), so this angular range is omitted from our analysis of LISST-VSF measurements. Note also that $C F_{400}(\psi)$ differs greatly from $C F_{100}(\psi)$ and $C F_{200}(\psi)$ within the angular range between about $65^{\circ}$ and $120^{\circ}$. This can be attributed to the uncertainty in the determinations of $C F_{400}(\psi)$ associated with a well-pronounced minimum in the volume scattering function for the $400 \mathrm{~nm}$ beads in this angular range. Therefore, the $C F_{400}(\psi)$ data in this angular range were not used in the determination of final $C F_{f}(\psi)$.

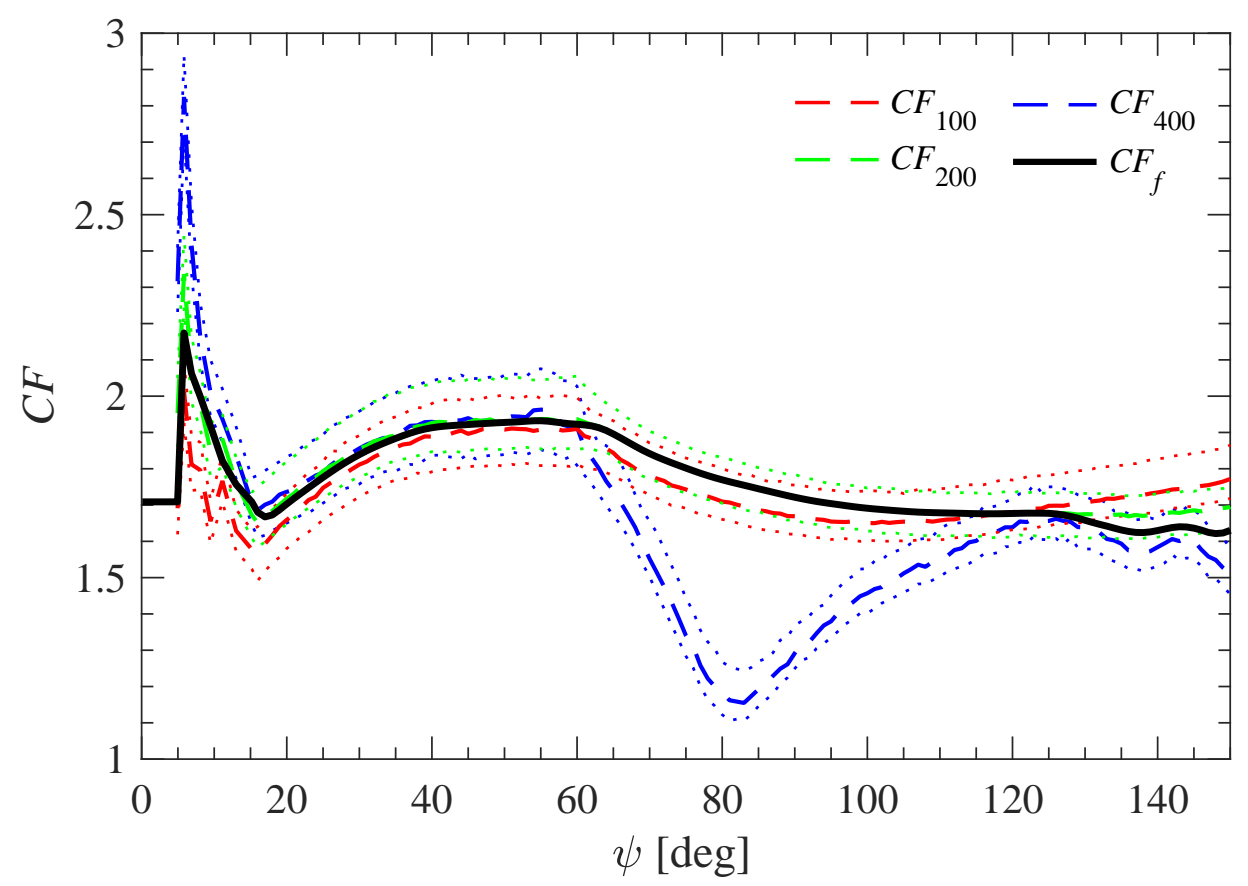

Figure 4. Correction functions, $C F(\psi)$, for the LISST-VSF measurements of particulate volume scattering function $\beta_{p}^{L I S S T *}(\psi)$ over the angular range $4.96-150^{\circ}$ determined for 100,200 , and $400 \mathrm{~nm}$ polystyrene bead suspensions. For each individual bead size, dashed lines represent the median values and the dotted lines indicate the 25th and 75th percentiles determined from the series of measurements. The final computed correction function $C F_{f}(\psi)$ is shown in black, and includes the constant value used for the near-forward angular range from $0.09^{\circ}$ to $4.96^{\circ}$.

The results for $B F_{100}(\psi), B F_{200}(\psi), B F_{400}(\psi)$, and $B F_{f}(\psi)$, are shown in Figure 5. As seen, $B F_{f}(\psi)$ is negative within the examined angular range and varies within a relatively narrow range of values between about -0.02 and -0.04 . Similar to the results for $C F_{400}(\psi)$, the distinct feature of positive bias observed in the $B F_{400}(\psi)$ data around the scattering angle of $80^{\circ}$ can be attributed to the uncertainty associated with a minimum in the volume scattering function for the $400 \mathrm{~nm}$ beads in this angular range. This portion of $B F_{400}(\psi)$ data was not used in the determination of final $B F_{f}(\psi)$.

The performance of the final correction function $C F_{f}(\psi)$ within the range of scattering angles from $0.09^{\circ}$ to $150^{\circ}$ was evaluated by comparing the corrected LISST-VSF measurements of volume scattering function, $\beta_{p}^{L I S S T}(\psi)$, with reference values of $\beta_{p}^{R E F}(\psi)$ for six samples of polystyrene beads $(100,200,400,500,700$, and $2000 \mathrm{~nm}$ in diameter; see the dilution factors for these samples indicated in boldface in Table 1). The beads with diameters of 500, 700, and $2000 \mathrm{~nm}$ were not used in the generation of the final correction function, so they provide completely independent data for evaluating the performance of $C F_{f}(\psi)$. The evaluation with the data for 100,200, and $400 \mathrm{~nm}$ beads is also useful because the final $C F_{f}(\psi)$ was determined by averaging the results obtained with multiple bead sizes and concentrations of these samples, and not from a single bead size and concentration. Results of independent measurements obtained with the DAWN-EOS on four bead suspensions $(100,200,400$, and $700 \mathrm{~nm}$ ) are also included in the evaluation analysis for additional comparisons. 


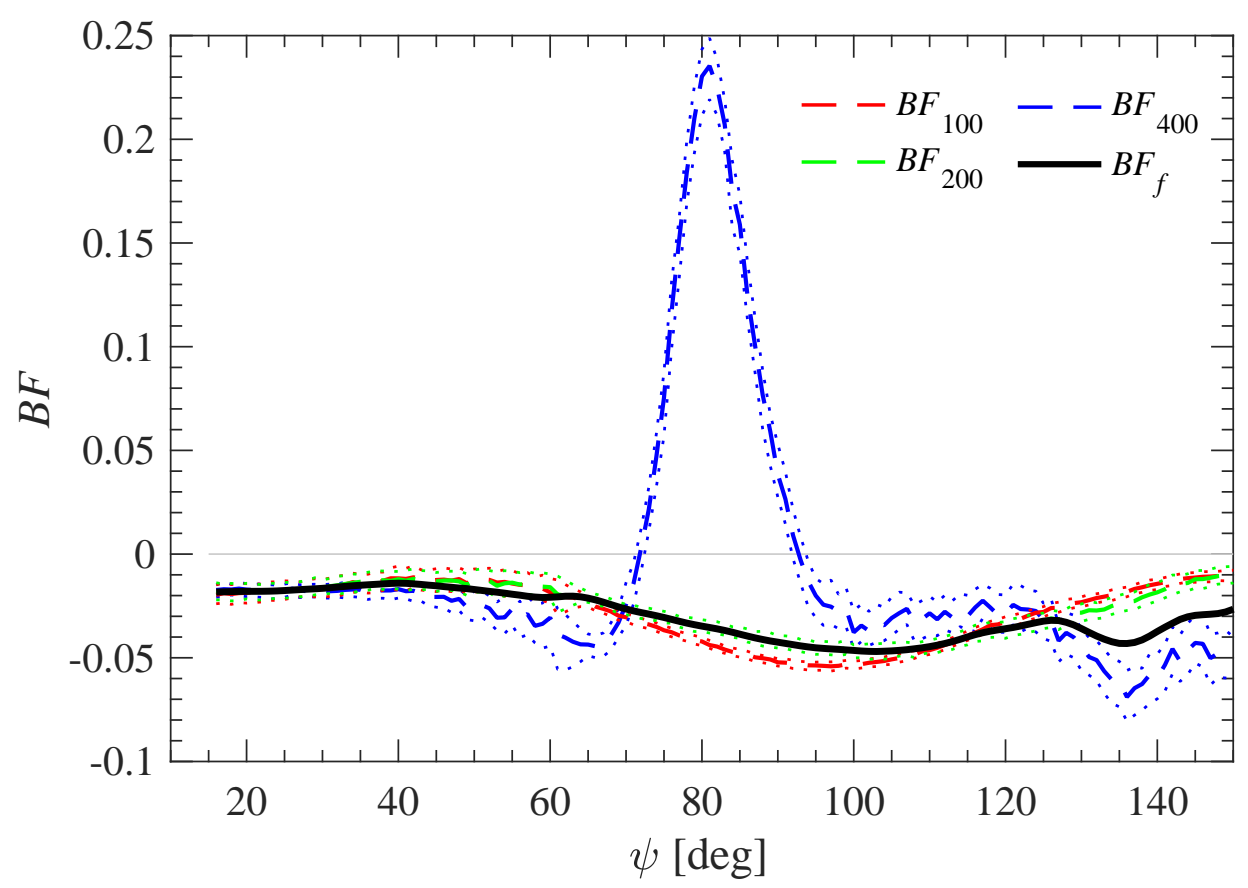

Figure 5. Correction functions, $B F(\psi)$, for LISST-VSF measurements of the degree of linear polarization of light scattered by particles, $\operatorname{DoLP}_{p}^{\operatorname{LISST} *}(\psi)$ over the angular range 16-150 determined for 100, 200, and $400 \mathrm{~nm}$ polystyrene bead suspensions. For each individual bead size, dashed lines represent the median values and the dotted lines indicate the 25th and 75th percentiles determined from the series of measurements. The final computed correction function $B F_{f}(\psi)$ is shown in black.

The comparisons of $\beta_{p}^{L I S S T}(\psi)$ and $\beta_{p}^{R E F}(\psi)$ are shown in Figure 6 for the six polystyrene bead samples. The presented values of $\beta_{p}^{\text {LISST }}(\psi)$ are the median values for each angle from each measurement series. The measured values of $\beta_{p}^{\mathrm{DAWN}}(\psi)$ are additionally depicted for the $100,200,400$, and $700 \mathrm{~nm}$ diameter beads. In general, the magnitude and angular dependence of $\beta_{p}^{L I S S T}(\psi)$ exhibits good agreement with reference values for all bead diameters. Notable differences occur within the minima of volume scattering function, for example near the angle of $80^{\circ}$ for the $400 \mathrm{~nm}$ beads (Figure $6 \mathrm{c}$ ). This issue has been mentioned above in the context of determinations of $C F_{f}(\psi)$ and $B F_{f}(\psi)$. The agreement observed between $\beta_{p}^{L I S T}(\psi)$ and $\beta_{p}^{\mathrm{DAWN}}(\psi)$ lends additional credence to the determined correction function $C F_{f}(\psi)$ and its application to LISST-VSF measurements.

Figure 7a illustrates the relationship between $\beta_{p}^{\operatorname{LISST}}(\psi)$ measured at all angles between $3.02^{\circ}$ and $150^{\circ}$ and $\beta_{p}^{R E F}(\psi)$ for the corresponding angles for the six bead samples. The overall agreement is quite good over a range spanning nearly 4 orders of magnitude. The regions of largest disagreement correspond to angles measured with the ring detectors, as well as angles corresponding to sharp minima or maxima in volume scattering function which are observed for the larger beads. Although the measured minima and maxima occur essentially at the same angles as predicted by Mie scattering calculations, the measured magnitude of minima or maxima can differ by a few tens of percent from the reference values. This is illustrated by plots of percent differences between the measured and reference values (Figure $7 \mathrm{~b}$ ). The oscillations and peaks (both positive and negative) in percent differences correspond to the minima and maxima in the angular patterns of volume scattering function. 

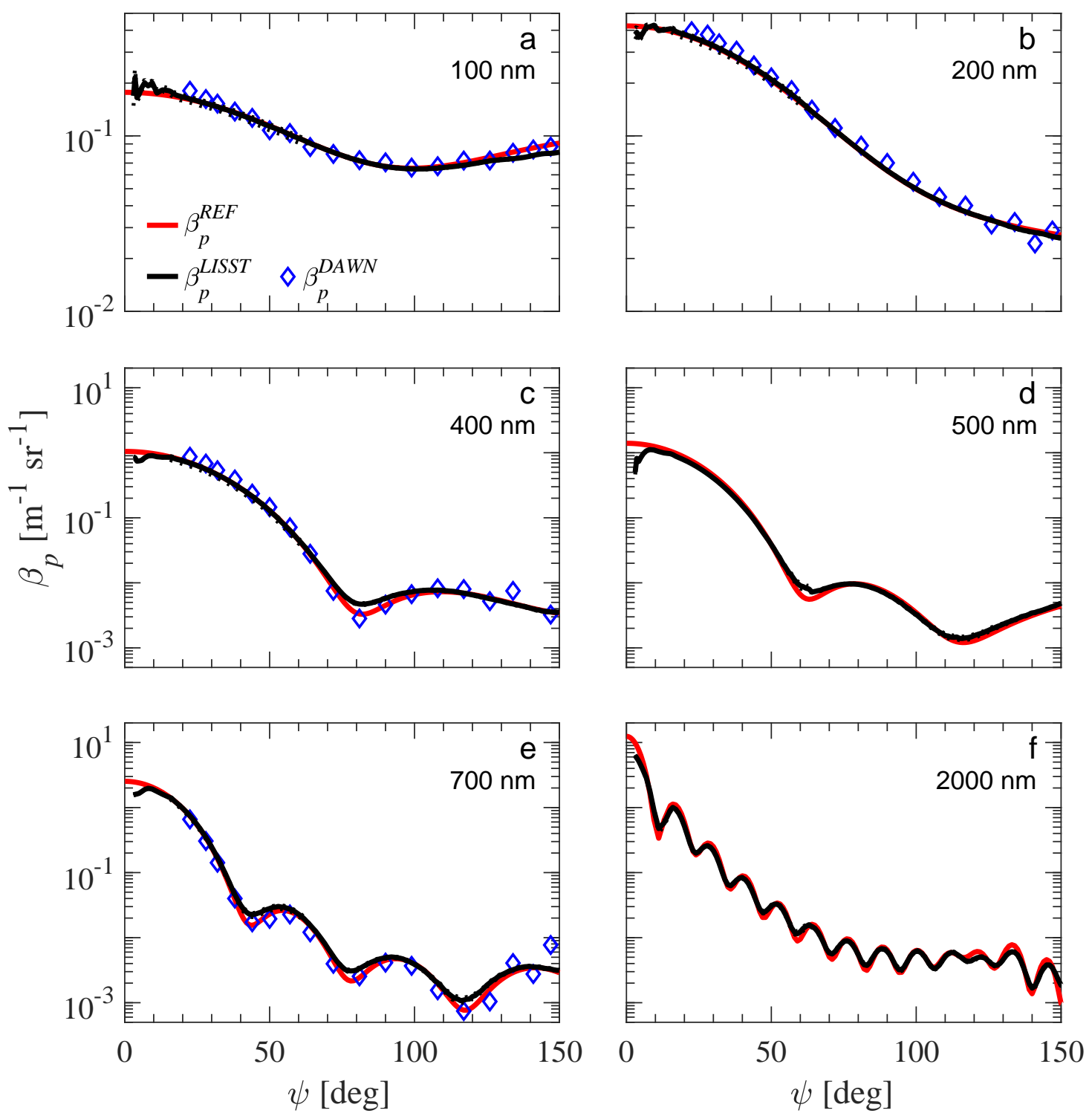

Figure 6. Comparison of $\beta_{p}(\psi)$ measurements on suspensions of polystyrene beads of varying diameter with reference values, $\beta_{p}^{R E F}(\psi)$. The $\beta_{p}^{L I S S T}(\psi)$ data represent $C F_{f}$-corrected median values obtained from a series of measurements with the LISST-VSF. Independent measurements of $\beta_{p}(\psi)$ obtained with the DAWN-EOS instrument are also shown as diamonds in panels a, b, c and e. The bead diameters are indicated in the legend.

Table 2 includes several statistical parameters that quantify the agreement between the data of $\beta_{p}^{L I S S T}(\psi)$ and $\beta_{p}^{R E F}(\psi)$ illustrated in Figure 7a. In this analysis we ignore $\psi<3.02^{\circ}$ due to generally low scattering signal relative to baseline for these ring detectors. The values of statistical parameters support the overall good agreement; for example, the median ratio $(M R)$ of $\beta_{p}^{L I S S T}(\psi)$ to $\beta_{p}^{R E F}(\psi)$ is very close to 1 , and the median absolute percent difference (MAPD) between $\beta_{p}^{L I S S T}(\psi)$ and $\beta_{p}^{R E F}(\psi)$ is only $\sim 4 \%$. These median values indicate no overall bias in the corrected measurements of $\beta_{p}^{\text {LISST }}(\psi)$ relative to the reference values of $\beta_{p}^{R E F}(\psi)$ and small statistical differences between $\beta_{p}^{\operatorname{LISST}}(\psi)$ and $\beta_{p}^{R E F}(\psi)$. Table 2 also includes the statistical parameters for a subset of data presented in Figure $7 \mathrm{a}$. In this subset, the forward scattering measurements with ring detectors were excluded, so the angular range is $16-150^{\circ}$. The statistical parameters for this subset are generally improved compared with the dataset covering the angular range $3.02-150^{\circ}$. For example, the root mean square difference $(R M S D)$ is smaller $\left(0.015 \mathrm{~m}^{-1} \mathrm{sr}^{-1}\right.$ vs. $\left.0.21 \mathrm{~m}^{-1} \mathrm{sr}^{-1}\right)$ and the slope of linear regression is closer to $1(0.958 \mathrm{vs}$. 
0.723). The improvements in the statistical parameters after removing the ring detector data are related primarily to much larger values of volume scattering function at forward scattering angles compared with larger angles, and a tendency to negative bias in $\beta_{p}^{L I S S T}(\psi)$ relative to $\beta_{p}^{R E F}(\psi)$ at forward angles.
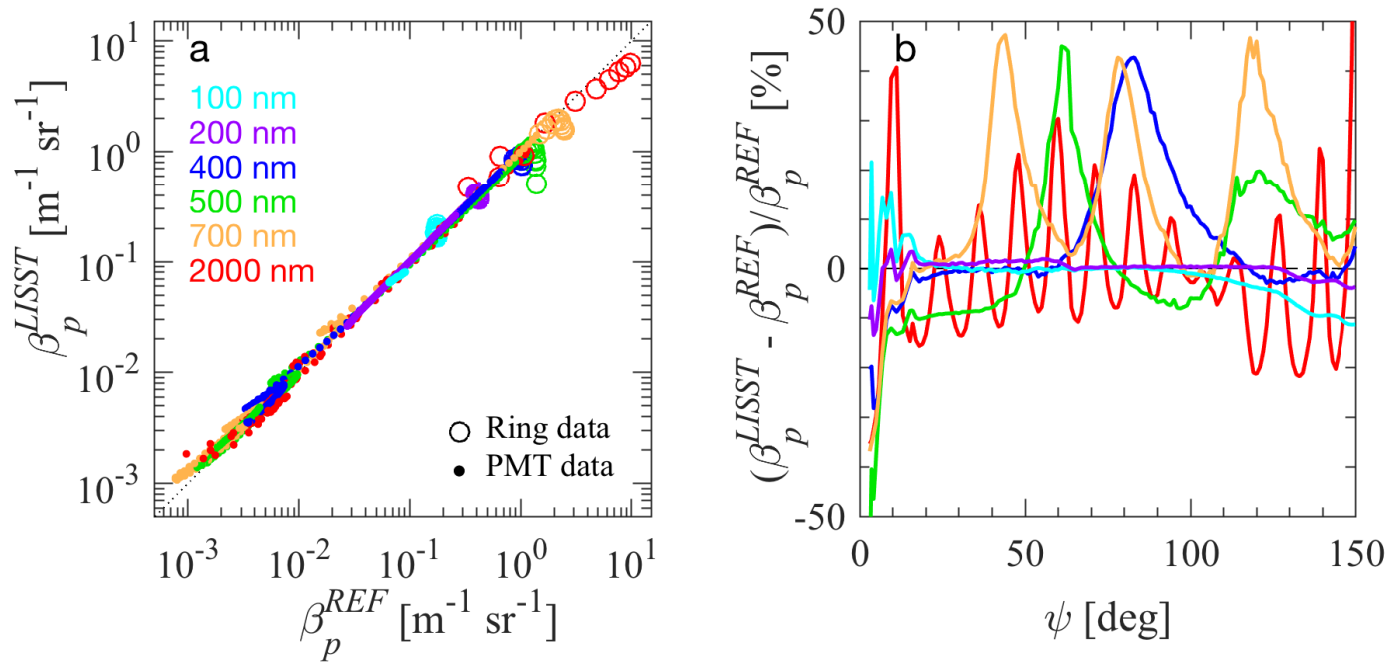

Figure 7. (a) Scatter plot of $\beta_{p}^{L I S S T}$ vs. $\beta_{p}^{R E F}$ for polystyrene beads of varying diameters as indicated. Data obtained with the ring detectors and Roving Eyeball sensor are plotted separately, and the 1:1 line is plotted for reference (dotted black line). (b) Residuals expressed as percentages between $\beta_{p}^{L I S S T}$ and $\beta_{p}^{R E F}$ for each bead size as a function of scattering angle.

To further validate the correction of LISST-VSF measurements with the $C F_{f}(\psi)$ function, we performed comparisons for approximate scattering and backscattering coefficients, $b_{p, 150}$ and $b_{b p, 150}$, respectively. The approximate scattering coefficient $b_{p, 150}$ was obtained by integrating the volume scattering function within the angular range from $0.09^{\circ}$ to $150^{\circ}$. The approximate backscattering coefficient $b_{b p, 150}$ was obtained by the integration from $90^{\circ}$ to $150^{\circ}$. These calculations were made for the uncorrected measured $\beta_{p}^{\operatorname{LISTT} *}(\psi), C F_{f}$-corrected measured $\beta_{p}^{\operatorname{LISST}}(\psi)$, and reference $\beta_{p}^{R E F}(\psi)$. We also used Mie scattering calculations to estimate the underestimation of the scattering and backscattering coefficients for the examined polystyrene beads caused by the integration of $\beta_{p}^{R E F}(\psi)$ up to $150^{\circ}$ as opposed to $180^{\circ}$. We found that the approximate scattering coefficient, $b_{p, 150}^{R E F}$, can be lower by as much as 7\% compared with the "true" scattering coefficient $b_{p}^{R E F}$. This result was observed for $100 \mathrm{~nm}$ beads. For backscattering the approximate coefficient $b_{b p, 150}^{R E F}$ was found to be lower by as much as $24 \%$ for the $500 \mathrm{~nm}$ beads. Although the LISST-VSF measurements extend to $150^{\circ}$ rather than $180^{\circ}$, the approximate coefficients are still useful for our validation exercise because most of the angular range and magnitude of total scattering and backscattering coefficients are included in the integration up to $150^{\circ}$. In addition, this validation analysis includes all 20 experiments conducted in this study, and not just the six example experiments presented in Figures 6 and 7.

Figure 8 compares the reference values of $b_{p, 150}^{R E F}$ and $b_{b p, 150}^{R E F}$ with LISST-VSF values determined from uncorrected $\beta_{p}^{\text {LISST* }}(\psi)$ and $C F_{f}$-corrected $\beta_{p}^{\operatorname{LISST}}(\psi)$. In these calculations we used the median values of $\beta_{p}^{L I S S T *}(\psi)$ and $\beta_{p}^{L I S S T}(\psi)$ for each measurement series from all 20 experimental combinations of bead sizes, concentrations, and PMT gains listed in Table 1. For all experiments, the approximate coefficients, $b_{p, 150}^{L I S T *}$ and $b_{b p, 150}^{L I S T *}$, derived from uncorrected $\beta_{p}^{L I S S T *}(\psi)$ are nearly half of the reference values of $b_{p, 150}^{R E F}$ and $b_{b p, 150}^{R E F}$. After $C F_{f}(\psi)$ correction the approximate coefficients $b_{p, 150}^{L I S T}$ and $b_{b p, 150}^{L L S T}$ exhibit a nearly 1:1 relationship with $b_{p, 150}^{R E F}$ and $b_{b p, 150}^{R E F}$. The statistical parameters that quantify the overall good agreement between $b_{p, 150}^{L I S S T}$ and $b_{p, 150}^{R E F}$ and between $b_{b p, 150}^{L I S S T}$ and $b_{b p, 150}^{R E F}$ are listed in Table 2 . 
Table 2. Statistical results evaluating the comparison of corrected data from the LISST-VSF measurements with reference values obtained from Mie scattering calculations. For $\beta_{p}^{L I S S T}$, the results are shown for the angular range $3.02-150^{\circ}$ which includes the ring data and for the range $16-150^{\circ}$ without the ring data. $R$ is the Pearson correlation coefficient and the coefficients $A$ and $B$ are the slope and $y$-intercept, respectively, determined from a type II linear regression between individual pairs of $X_{i}$ and $Y_{i}$ values where $Y_{i}$ represents measured values and $X_{i}$ reference values. The mean bias $(M B)$ was calculated as $1 / N \times \sum_{i=1}^{N}\left(Y_{i}-X_{i}\right)$ and $M R$ represents the median ratio of $Y_{i} / X_{i}$. The root mean squared deviation, $R M S D$, was calculated as $\sqrt{\frac{1}{N} \sum_{i=1}^{N}\left(Y_{i}-X_{i}\right)^{2}}$, and the median absolute percent difference, $M A P D$, was calculated as the median value of $\left|\frac{Y_{i}-X_{i}}{X_{i}}\right| \times 100 . N$ is the number of data points used in the analysis.

\begin{tabular}{lcccccccc}
\hline \multicolumn{1}{c}{ Data } & $\boldsymbol{R}$ & $\boldsymbol{A}$ & \multicolumn{1}{c}{$\boldsymbol{B}$} & \multicolumn{1}{c}{$\boldsymbol{M B}$} & MR & \multicolumn{1}{c}{ RMSD } & MAPD & N \\
\hline$\beta_{p}^{\text {LISST }}(\mathrm{w} / \mathrm{rings})$ & 0.987 & 0.72 & $0.031 \mathrm{~m}^{-1} \mathrm{sr}^{-1}$ & $-0.028 \mathrm{~m}^{-1} \mathrm{sr}^{-1}$ & 1.00 & $0.210 \mathrm{~m}^{-1} \mathrm{sr}^{-1}$ & $3.94 \%$ & 876 \\
$\beta_{p}^{\text {LISST }}$ (w/o rings) & 0.998 & 0.96 & $0.002 \mathrm{~m}^{-1} \mathrm{sr}^{-1}$ & $-0.002 \mathrm{~m}^{-1} \mathrm{sr}^{-1}$ & 1.00 & $0.015 \mathrm{~m}^{-1} \mathrm{sr}^{-1}$ & $3.39 \%$ & 810 \\
$b_{p, 150}^{\text {LISST }}$ & 0.995 & 1.04 & $-0.048 \mathrm{~m}^{-1}$ & $-0.007 \mathrm{~m}^{-1}$ & 1.00 & $0.043 \mathrm{~m}^{-1}$ & $2.30 \%$ & 20 \\
$b_{b p, 150}^{\text {LIST }}$ & 0.999 & 0.99 & $0.0001 \mathrm{~m}^{-1}$ & $-0.001 \mathrm{~m}^{-1}$ & 1.00 & $0.006 \mathrm{~m}^{-1}$ & $3.70 \%$ & 20 \\
$\operatorname{DoLP}_{p}^{\text {LISST }}$ & 0.989 & 0.91 & 0.046 & 0.016 & 0.99 & 0.065 & $5.00 \%$ & 810 \\
\hline
\end{tabular}
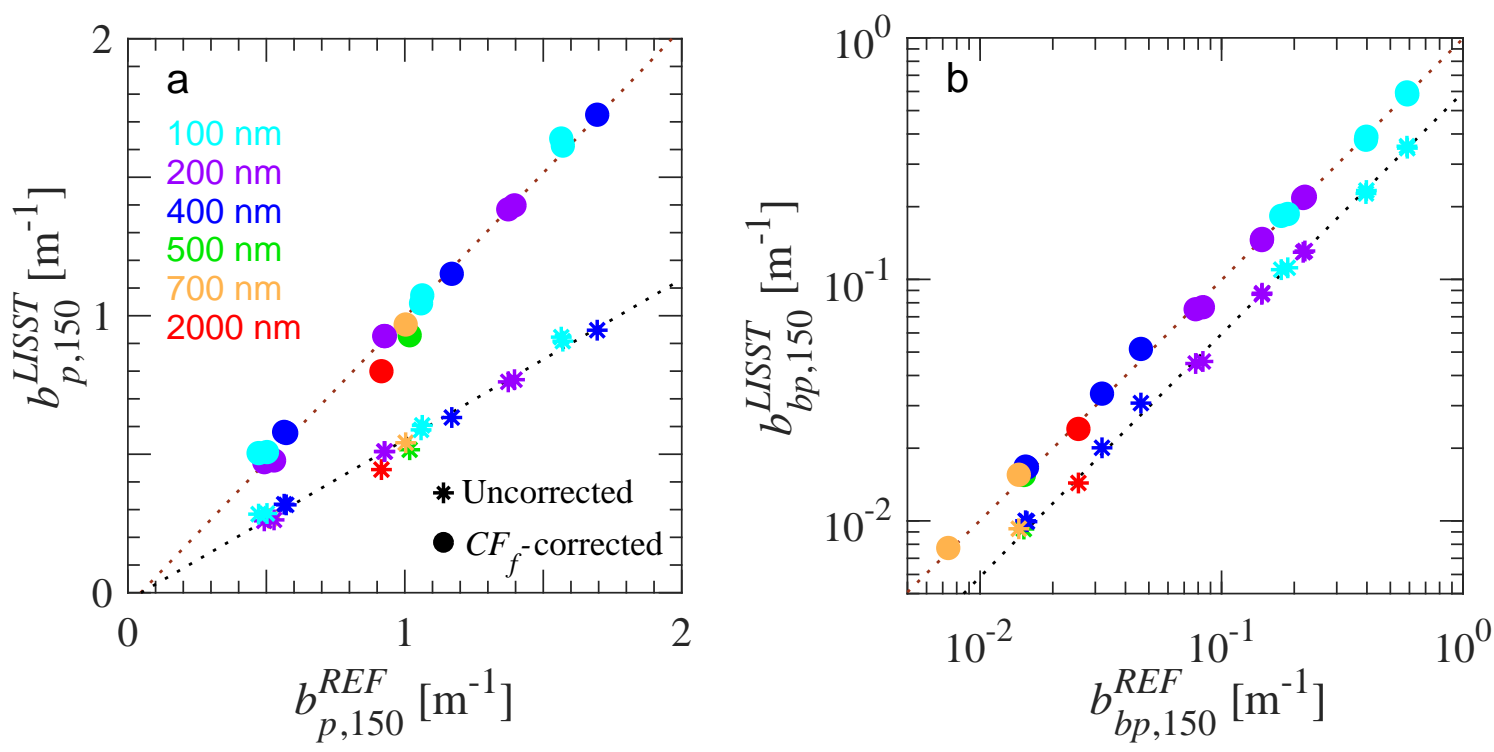

Figure 8. (a) Scatter plot comparing reference values of the particulate scattering coefficient computed over the angular range $0.09-150^{\circ}, b_{p, 150}^{R E F}$, with values determined from the LISST-VSF, $b_{p, 150}^{L I S S T}$, before (asterisks) and after (circles) correction with $C F_{f}$. A type II linear regression model fit to the data is indicated by the dotted lines. (b) Similar to (a), but for the particulate backscattering coefficient computed over the range $90-150^{\circ}$.

Similarly to the validation analysis of $C F_{f}(\psi)$, the performance of the correction function $B F_{f}(\psi)$ was evaluated by comparing the corrected LISST-VSF measurements of the degree of linear polarization, $\operatorname{DoLP} P_{p}^{L I S S T}(\psi)$, with reference values of $\operatorname{DoLP} P_{p}^{R E F}(\psi)$ for six samples of polystyrene beads (100, 200, $400,500,700,2000 \mathrm{~nm}$ in diameter). Figure 9 depicts these comparisons. The values of $\operatorname{DoLP} P_{p}^{\mathrm{DAWN}}(\psi)$ measured with DAWN-EOS are also depicted for the 100, 200, 400, and $700 \mathrm{~nm}$ beads. For all bead sizes, the magnitude and angular dependence of $\operatorname{DoLP}_{p}^{L I S S T}(\psi)$ exhibits generally a very good agreement with both the reference values and DAWN-EOS measurements. For larger beads, notable differences occur within the minima of the degree of linear polarization, for example near the angle of $80^{\circ}$ for the $400 \mathrm{~nm}$ beads (Figure 9c). 

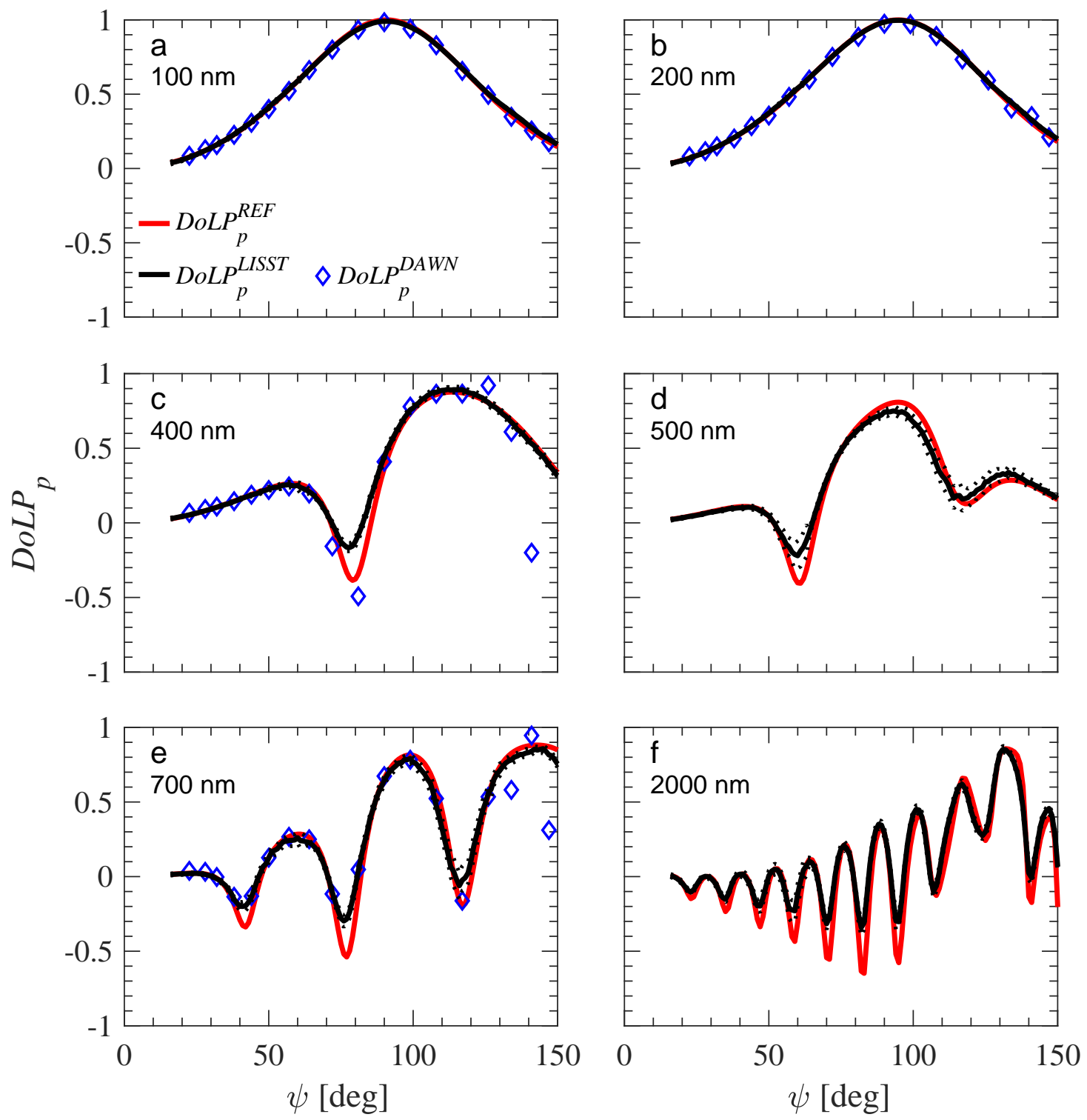

Figure 9. Similar to Figure 6, but for measured and reference values of particulate degree of linear polarization $\operatorname{DoLP}_{p}$. Measurements obtained with the LISST-VSF were corrected with $B F_{f}$.

Figure 10a is a scatter plot of $\operatorname{DoLP} P_{p}^{L I S S T}(\psi)$ vs. $\operatorname{DoLP} P_{p}^{R E F}(\psi)$ which includes all data for the six bead samples presented in Figure 9. In the region of negative values which correspond to the minima in the angular pattern of the degree of linear polarization, the $\operatorname{DoLP}_{p}^{L I S S T}(\psi)$ exhibits a positive bias relative to $\operatorname{DoLP} P_{p}^{R E F}(\psi)$. This bias is seen in the form of peaks in the angular pattern of the difference between $\operatorname{DoLP} P_{p}^{L I S S T}(\psi)$ and $\operatorname{DoLP} P_{p}^{R E F}(\psi)$ for larger bead sizes (Figure 10b). The peak amplitudes generally range from 0.05 to 0.3. Importantly, however, aside from these features the data of $\operatorname{DoLP}_{p}^{L I S S T}(\psi)$ vs. $\operatorname{DoLP}_{p}^{R E F}(\psi)$ are distributed close to the 1:1 line within the major part of the region of positive values (Figure 10a). This includes the region of maximum values of the degree of linear polarization of scattered light from natural seawater samples, which are observed at scattering angles near $90^{\circ}$ or greater. The overall good agreement between $B F_{f}$-corrected measured $\operatorname{DoL} P_{p}^{L I S T}(\psi)$ and $\operatorname{DoLP} P_{p}^{R E F}(\psi)$ is supported by the statistical parameters shown in Table 2 which are calculated on the basis of the entire dataset presented in Figure 10. For example, the RMSD and MAPD values are small, 0.065 and 5\%, respectively. Also, despite some negative bias for data with negative values of the degree of linear polarization, the $M R$ for the ratio of $\operatorname{DoLP}_{p}^{L I S S T}(\psi)$ to $\operatorname{DoLP}_{p}^{R E F}(\psi)$ for the entire dataset is 0.993 , indicating 
essentially no bias. These statistics would improve if the data within the minima in the angular pattern of the degree of linear polarization were removed from the analysis.
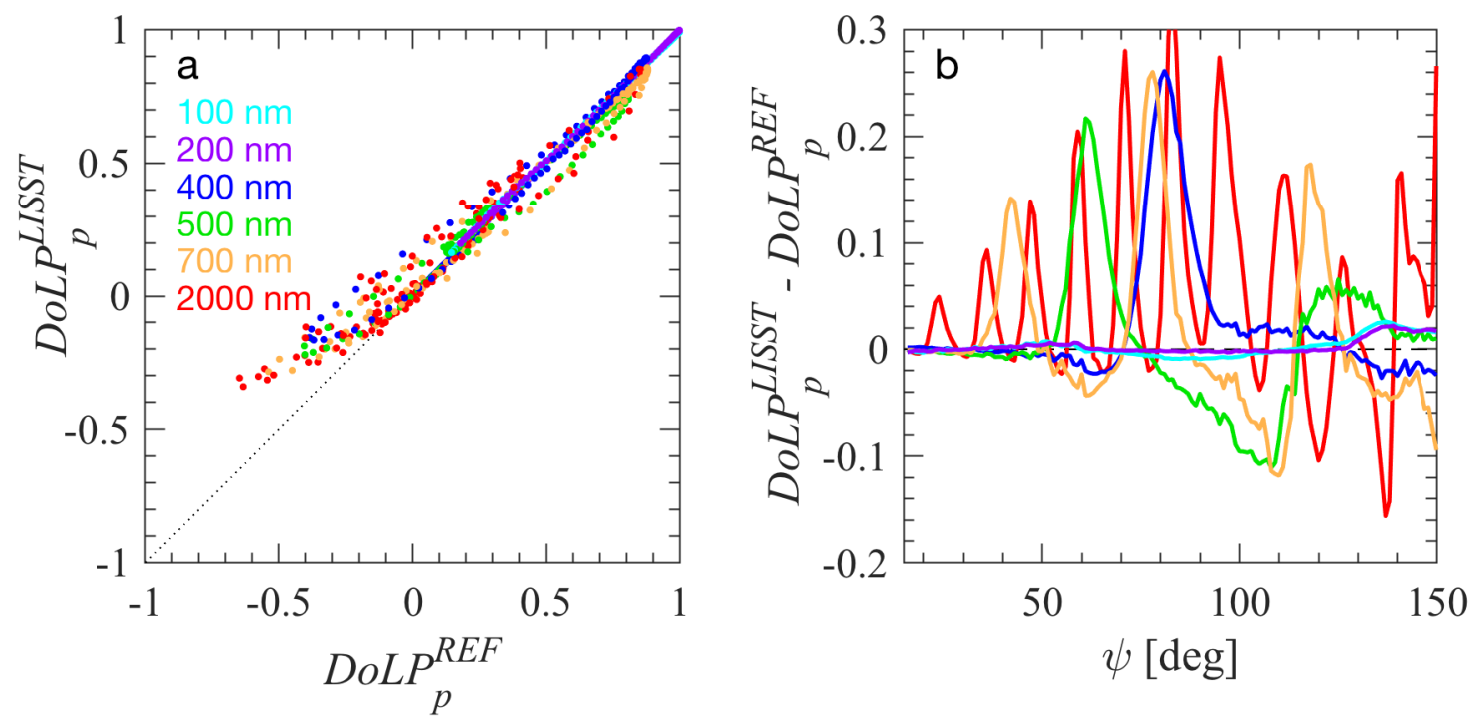

Figure 10. Similar to Figure 7, but for $D o L P_{p}$. All data are obtained with the Roving Eyeball sensor, and the residuals between $D o L P_{p}^{L I S S T}$ and $D o L P_{p}^{R E F}$ in $(\mathbf{b})$ are expressed as absolute differences.

\subsection{Measured Light Scattering Properties of Natural Particulate Assemblages}

Figure 11 depicts the corrected measured volume scattering function, $\beta_{p}^{\text {LISST }}(\psi)$, and the degree of linear polarization, $\operatorname{DoLP}_{p}^{L I S S T}(\psi)$, for three contrasting natural assemblages of particles. The selected parameters describing the particulate and optical properties of these samples are provided in Table 3 . Sample A was obtained $\sim 8 \mathrm{~km}$ offshore from the subsurface chlorophyll- $a$ maximum at a depth of $18 \mathrm{~m}$, sample B was collected just beneath the sea surface at SIO Pier during a calm sunny summer day, and sample $C$ was collected $\sim 2 \mathrm{~km}$ inland at the San Diego River Estuary during low tide. The SPM range covers about one order of magnitude from $0.36 \mathrm{~g} \mathrm{~m}^{-3}$ for sample A to $3.18 \mathrm{~g} \mathrm{~m}^{-3}$ for sample $\mathrm{C}$, which is reflected in significant range of the particulate scattering coefficient, $b_{p}^{L I S S T}$, from $0.36 \mathrm{~m}^{-1}$ to $2.23 \mathrm{~m}^{-1}$. Chla was also lowest for sample A $\left(0.75 \mathrm{mg} \mathrm{m}^{-3}\right)$ but highest for sample $\mathrm{B}\left(2.5 \mathrm{mg} \mathrm{m}^{-3}\right)$. Thus, whereas the offshore sample A represents a particle concentration that is within the range of observations in relatively clear open ocean waters, sample $C$ is representative of more turbid coastal or nearshore waters [5,7]. Samples A and B have similarly high values of the ratio POC/SPM (0.43 and 0.47, respectively) and relatively high values of Chla/SPM $\left(2.1 \times 10^{-3}\right.$ and $2.2 \times 10^{-3}$, respectively), indicating organic-dominated particulate assemblages with significant contribution of phytoplankton. In contrast, sample $C$ has much lower values of POC/SPM (0.14) and Chla/SPM $\left(3.8 \times 10^{-4}\right)$, indicating inorganic-dominated particulate assemblage and relatively small role of phytoplankton, despite significant chlorophyll-a concentration $\left(1.21 \mathrm{mg} \mathrm{m}^{-3}\right)$.

These differences in particle properties between the three samples are responsible for the differences in the magnitude and angular shape of $\beta_{p}^{L I S S T}(\psi)$ and $\operatorname{DoLP}_{p}^{L I S S T}(\psi)$ presented in Figure 11 and the optical parameters listed in Table 3. These optical parameters include the particulate backscattering ratio, $\widetilde{b}_{b p}^{\text {LISST }}$, the ratio of $\beta_{p}^{\text {LISST }}\left(45^{\circ}\right)$ to $\beta_{p}^{\text {LISST }}\left(135^{\circ}\right)$, and the maximum value of $\operatorname{DoLP}_{p}^{\text {LISST }}(\psi)$ denoted as $D o L P_{p, \max }^{L I S T}$. This maximum value occurs at a scattering angle $\psi_{\max }$ which is also provided in Table 3. The offshore sample A has an intermediate value of $\widetilde{b}_{b p}^{L I S S T}$ and the highest DoLP $P_{p, \max }^{L I S T}$ of about 0.77 associated with the smallest $\psi_{\max }$ of $92^{\circ}$. Sample B from the SIO Pier has the lowest $\widetilde{b}_{b p}^{\text {LISST }}$ of 0.008 among the three samples, suggesting a relatively steep slope of particle size distribution, relatively low bulk particle refractive index, or both [17]. This sample also shows the least steep near-forward scattering pattern (Figure 11c), which suggests a higher proportion of small particles relative to larger 
particles compared with the two other samples. Finally, the most turbid and least organic sample, sample $C$, exhibits an enhanced proportion of backscattering with the highest $\widetilde{b}_{b p}^{\text {LISST }}$ of 0.027 . While this result may suggest a relatively high bulk particle refractive index [17] consistent with the lowest POC/SPM ratio among the three samples, the additional influence of particle size distribution cannot be ruled out. Sample C shows steep near-forward scattering pattern (Figure 11c), which is typically indicative of an increased proportion of large particles relative to small particles. Note that sample $C$ also has the lowest $\beta_{p}^{L I S S T}\left(45^{\circ}\right) / \beta_{p}^{L I S S T}\left(135^{\circ}\right)$ ratio of 12 , which indicates a higher degree of symmetry in the angular pattern of scattering about $90^{\circ}$, which is consistent with the relatively high value of $\widetilde{b}_{b p}^{\text {LISST }}$ for this sample. In addition, sample C has the lowest DoLP $P_{p, \max }^{\mathrm{LIST}}$ of 0.58 .
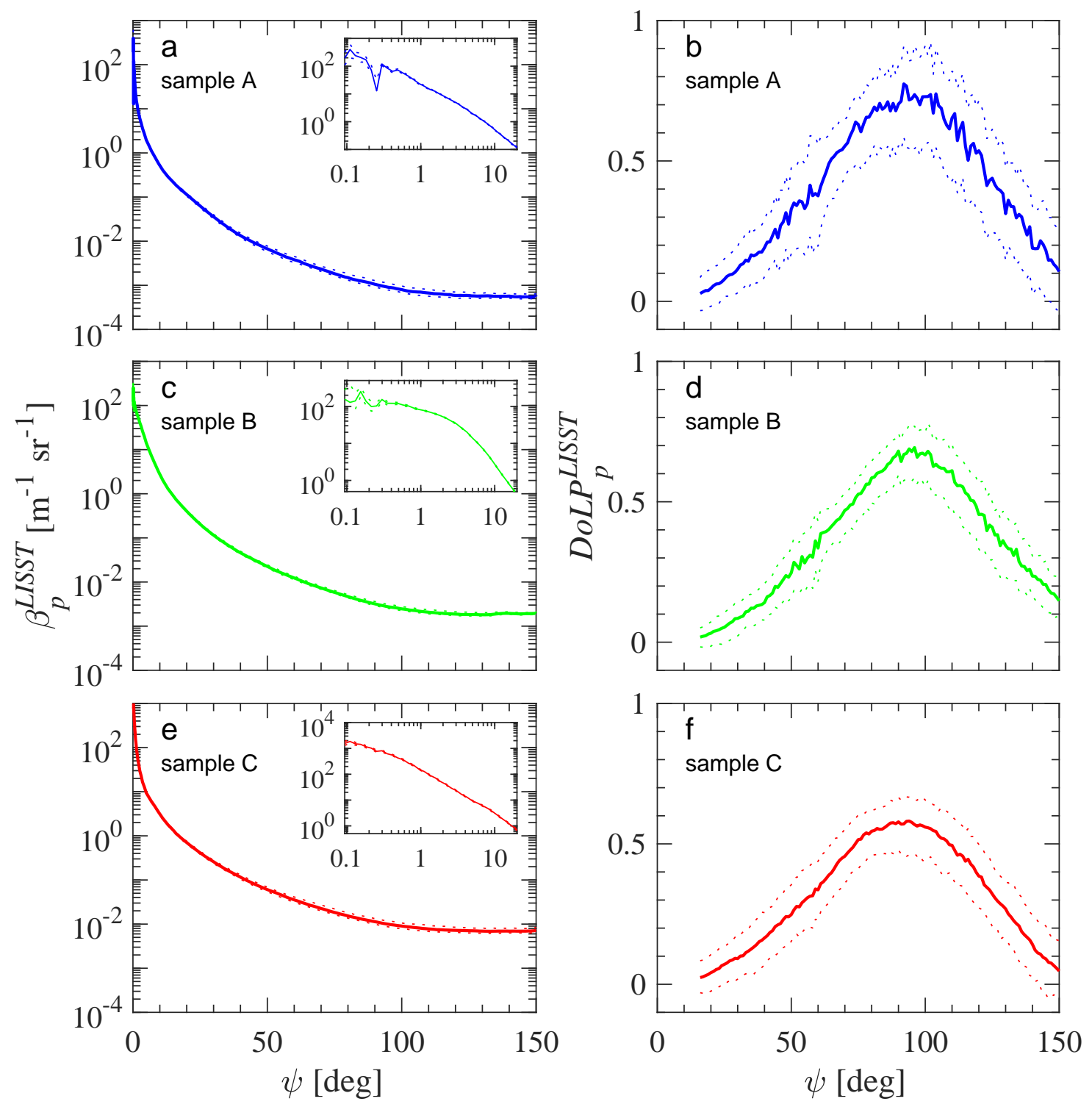

Figure 11. Measurements of $\beta_{p}^{L I S S T}$ and DoLP $P_{p}^{L I S S T}$ obtained with the LISST-VSF on natural seawater samples from the San Diego region representing $(\mathbf{a}, \mathbf{b})$ subsurface offshore waters, (c,d) SIO Pier, and $(\mathbf{e}, \mathbf{f})$ San Diego River Estuary. Solid lines represent median values while dotted lines indicate the $10^{\text {th }}$ and $90^{\text {th }}$ percentiles obtained from a series of measurements on each sample. Insets in $(\mathbf{a}, \mathbf{c}, \mathbf{d})$ display greater detail on the near-forward scattering range. 
Table 3. General information on particle characteristics and median values of optical quantities derived from LISST-VSF measurements for the three example natural seawater samples depicted in Figure 11. Values of selected optical quantities estimated from the measurements of Petzold [38] are shown for comparison.

\begin{tabular}{|c|c|c|c|c|c|c|c|c|}
\hline $\begin{array}{c}\text { Sample } \\
\text { ID }\end{array}$ & $\begin{array}{c}\text { Chla } \\
{\left[\mathrm{mg} \mathrm{m}^{-3}\right]}\end{array}$ & $\begin{array}{c}\text { SPM } \\
{\left[\mathrm{g} \mathrm{m}^{-3}\right]}\end{array}$ & $\begin{array}{c}\text { POC/SPM } \\
{[\mathrm{g} / \mathrm{g}]}\end{array}$ & $\begin{array}{c}b_{p} \\
{[m-1]}\end{array}$ & $\begin{array}{c}\widetilde{b}_{b p} \\
{[\mathrm{dim}]}\end{array}$ & $\frac{\beta_{p}\left(45^{\circ}\right)}{\beta_{p}\left(135^{\circ}\right)}$ & $\begin{array}{c}\operatorname{DoLP} P_{p, \max } \\
\quad[\mathrm{dim}]\end{array}$ & $\begin{array}{r}\psi_{\max } \\
{[\mathrm{deg}]}\end{array}$ \\
\hline $\mathrm{A}$ & 0.75 & 0.36 & 0.43 & 0.36 & 0.012 & 16.5 & 0.77 & 92 \\
\hline B & 2.49 & 1.13 & 0.47 & 1.75 & 0.008 & 17.1 & 0.69 & 96 \\
\hline C & 1.21 & 3.18 & 0.14 & 2.23 & 0.022 & 12.0 & 0.58 & 94 \\
\hline \multicolumn{9}{|c|}{ Petzold Measurements } \\
\hline Clear & & & & 0.03 & 0.015 & 18.0 & & \\
\hline Coastal & & & & 0.19 & 0.009 & 17.8 & & \\
\hline Turbid & & & & 1.74 & 0.020 & 12.2 & & \\
\hline
\end{tabular}

For comparison, Table 3 also includes the values for the selected optical parameters estimated from measurements reported by Petzold [38] for clear ocean waters (off Bahamas), coastal waters (San Diego coastal region), and turbid waters (San Diego Harbor). These measurements span a generally similar range of scattering angles as the LISST-VSF (10-180 in $5^{\circ}$ increments), but are based on a spectrally broader incident beam (75 $\mathrm{nm}$ full width half maximum) centered at $514 \mathrm{~nm}$. The estimates of particulate volume scattering function $\beta_{p}$ from Petzold's measurements were obtained by subtracting pure seawater contribution $\beta_{w}$ from the measured total $\beta$. The $\beta_{w}$ value was calculated assuming a water temperature of $15{ }^{\circ} \mathrm{C}$ and salinity of 33 PSU [77]. Although Petzold's data include measurements made in clearer waters compared with our samples, the range of values for the dimensionless parameters associated with the shape of angular scattering pattern, $\widetilde{b}_{b p}^{\text {LISTT }}$ and $\beta_{p}^{L I S S T}\left(45^{\circ}\right) / \beta_{p}^{L I S S T}\left(135^{\circ}\right)$, is very similar. Specifically, our data for the offshore sample A are similar to Petzold's data from clear waters, sample B from the SIO Pier aligns with Petzold's data from coastal San Diego waters, and sample C from San Diego River Estuary with Petzold's data from the San Diego Harbor.

We note that the dotted lines in Figure 11 reflect some variations in $\beta_{p}^{L I S S T}(\psi)$ and $\operatorname{DoLP}_{p}^{L I S S T}(\psi)$ between the individual measurements within a given set of measurements for each sample. For example, on the basis of the collection of 200 to 400 measurements for each of the two linear polarization states of the incident beam and the scattered light, the coefficient of variation at $\psi=$ $90^{\circ}$ was $14 \%, 8 \%$, and $13 \%$ for $\beta_{p}^{L I S S T}$ and $20 \%, 13 \%$, and $15 \%$ for $D o L P_{p}^{L I S S T}$ for samples A, B, and C, respectively. These variations between the individual measurements that have been taken in rapid succession do not necessarily reflect the measurement precision, as they can also be associated with actual variations in the sample, for example the fluctuations in the presence of relatively rare large particles within the interrogated sample volume. Another important point is that the small negative values of DoLP $P_{p}^{L I S S T}$ observed for some individual measurements at forward scattering angles $<30^{\circ}$ (see the 10th percentile dotted lines in Figure 11b,d,f) are not necessarily an indication of measurement uncertainty because the negative values, especially in this angular range, are physically possible for certain types of particles $[30,54,56,58]$.

Figure 12 depicts scatter plots of the relationships between the dimensionless optical parameters, $\widetilde{b}_{b p}^{L I S S T}$ and DoLP $P_{p, \max }^{L I S T}$, and the dimensionless particulate compositional properties, POC/SPM and Chla/SPM, for all 17 samples examined in this study. The overall range of POC/SPM in our dataset is 0.04 to 0.6. The presented data have been divided into three groups according to the values of POC/SPM as follows: the least organic-dominated (or the most mineral-dominated) data with $\mathrm{POC} / \mathrm{SPM}<0.15$; the most organic-dominated data with POC $/ \mathrm{SPM}>0.3$, and the intermediate data with $0.15 \leq \mathrm{POC} / \mathrm{SPM} \leq 0.3$. The selected boundary values of POC/SPM for discriminating between the organic-dominated and mineral-dominated groups of data differ from those used in our previous studies $[65,78]$, but appear to adequately reflect the patterns in the present data. In particular, the most mineral-dominated samples with POC/SPM $<0.15$ form a clear cluster of data points with the highest $\widetilde{b}_{b p}^{\text {LISST }}$ (Figure 12a) and the lowest Chla/SPM (Figure 12b,d). We also note that no data were collected 
for POC/SPM between 0.15 and 0.2 , so we will refer to all data with POC/SPM $>0.2$ as highly organic because they all represent highly significant or dominant role of organic particles.
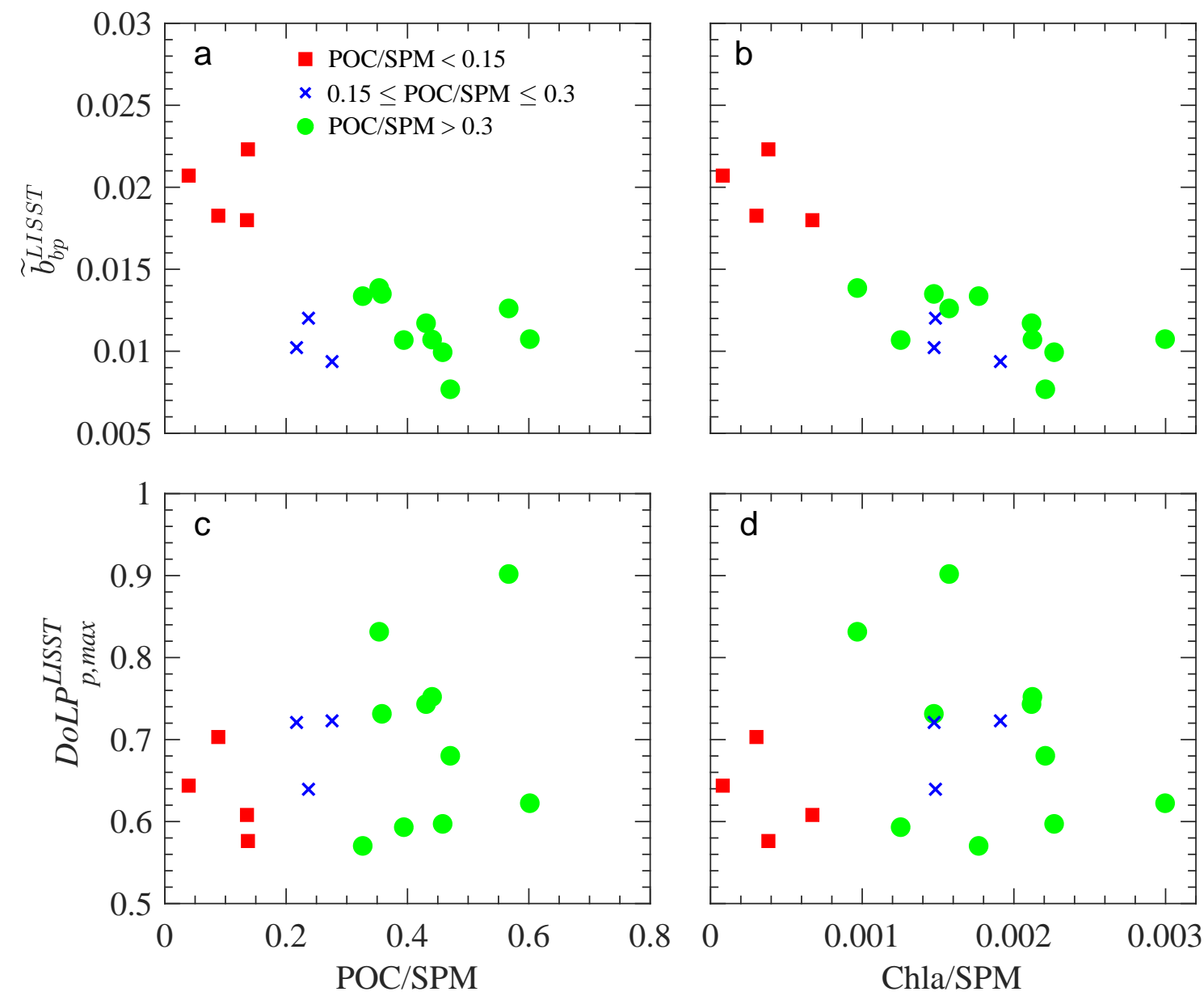

Figure 12. LISST-VSF measurements of $(\mathbf{a}, \mathbf{b})$ the particulate backscattering ratio, $\widetilde{b}_{b p}^{\text {LISST }}$, and $(\mathbf{c}, \mathbf{d})$ the maximum value of the degree of linear polarization of scattered light, DoLP $P_{p, \max }^{\mathrm{LIST}}$, as a function of the POC/SPM or Chla/SPM ratio. The data are divided into three groups defined by the range of POC/SPM as indicated in the legend.

The scatter plot for the data of $\widetilde{b}_{b p}^{L I S S T}$ vs. POC/SPM suggests the presence of a relational trend with significant negative correlation between the variables (the correlation coefficient $R=-0.73$ ). While the $\widetilde{b}_{b p}^{\text {LIST }}$ values are clearly highest for POC $/ \mathrm{SPM}<0.15$, the organic-dominated samples have consistently lower $\widetilde{b}_{b p}^{L I S S T}$. This result is consistent with the notion that mineral-dominated particulate assemblages with relatively high bulk refractive index of particles tend to have higher backscattering ratio $\widetilde{b}_{b p}$ compared with organic-dominated assemblages with lower refractive index [17]. However, we also note that highly organic samples with POC/SPM $>0.2$ show no clear relationship and essentially no correlation between $\widetilde{b}_{b p}^{L I S T}$ and POC/SPM $(R=-0.11)$. This result may be attributable to the effect of other particle characteristics on $\widetilde{b}_{b p}^{L I S S T}$, such as variations in refractive index of particles associated with changes in the composition of particulate organic matter, variations in particle size distribution, or both. The scatter plot of $\widetilde{b}_{b p}^{\text {LISST }}$ vs. Chla/SPM (Figure 12b) provides interesting insight into this question, as this relationship is significantly better compared with $\widetilde{b}_{b p}^{L S S T}$ vs. POC/SPM. Whereas the correlation between $\widetilde{b}_{b p}^{\text {LISST }}$ and Chla/SPM for all data is strong $(R=-0.85)$, the subset of data for highly organic samples (POC/SPM $>0.2$ ) has also a relatively high correlation coefficient of -0.51 . This is an important result, suggesting that for particulate assemblages with high organic content, 
the backscattering ratio $\widetilde{b}_{b p}$ tends to decrease with increasing proportion of phytoplankton in the particulate assemblage. It is likely that the relationship in Figure $12 b$ is largely driven by a decrease in the bulk particle refractive index with increasing proportion of phytoplankton in the particulate assemblage. Because this trend also holds for the subset of highly organic samples, it may indicate that live phytoplankton cells have generally lower refractive index than non-living organic particles.

In contrast to $\widetilde{b}_{b p}^{L I S T}$, the DoLP $P_{p, \max }^{L I S T}$ data show no clear relational trend and very weak correlation with POC/SPM $(R=0.31)$, indicating that the maximum degree of linear polarization does not provide a useful optical signature for the organic vs. inorganic content of particulate assemblages in our dataset (Figure 12c). A similar result with no correlation $(R=0.07)$ is observed for DoLP $P_{p, \max }^{\text {LIST }}$ vs. Chla/SPM, indicating that varying proportion of phytoplankton in total particulate assemblage has no discernible systematic effect on the maximum degree of linear polarization (Figure 12d). We also determined that there is no significant correlation between $D o L P_{p, \max }^{L I S T}$ and $\widetilde{b}_{b p}^{L I S S T}$ in our dataset $(R=-0.22)$, as well as between $\psi_{\max }$ and POC/SPM or Chla/SPM $(R=-0.09$ and 0.06 , respectively). It is also of interest to note that the range of our $D o L P P_{p, \max }^{L I S T}$ data is generally consistent with the range of values reported in literature for natural seawater samples, although the reported range in some earlier studies extends to somewhat lower values, as low as about $0.4[15,39-42,79,80]$.

The assessment of potential presence of systematic effects of particle size distribution (PSD) on $\widetilde{b}_{b p}^{L I S S T}$ and DoLP $P_{p, \max }^{\text {LIST }}$ is presented in Figure 13. In this assessment, we use two PSD metrics: the $90^{\text {th }}$ percentile diameter, $D_{V}^{90}$, derived from the particle volume distribution, and the power function slope, $\zeta$, derived from the particle number distribution. We also tested other percentile-based diameters such as the median $D_{V}^{50}$ but no improvements in the examined relationships were observed. Figure 13a,b shows no trend in the data of $\widetilde{b}_{b p}^{L I S S T}$ associated with variations in the particle size metrics, even though these metrics vary over a significant dynamic range. This is the case for the entire dataset as well as a subset of highly organic samples with POC/SPM $>0.2$, which supports the interpretation of results presented in Figure $12 \mathrm{~b}$ in terms of the role of refractive index. The data of $D o L P_{p, \max }^{L I S T} \mathrm{vs} . D_{V}^{90}$ show the potential for the presence of a relational trend (Figure 13c). Although the scatter in these data points is significant and correlation is weak $(R=-0.47)$, the lowest values of $D o L P_{p \text {, } \max }^{L S S T}$ tend to occur along with the highest values of $D_{V}^{90}$. This result indicates that the decrease in the maximum degree of linear polarization tends to be associated with particulate assemblages exhibiting a higher proportion of large-sized particles. The potential usefulness of the relationship between the degree of linear polarization and particle size has been proposed for the first time in 1930 [81], and the trend observed in our data is consistent with those early results.

\section{Concluding Remarks}

Our laboratory measurements combined with Mie scattering calculations for samples of standard polystyrene beads illustrate the value of such an approach for evaluating the calibration and performance of light scattering instruments. For the specific version of LISST-VSF instrument and data processing code used in our study, we determined the calibration correction functions for improved determinations of the particulate volume scattering function $\beta_{p}(\psi)$ and the degree of linear polarization $\operatorname{DoLP}_{p}(\psi)$. The required correction was found to be particularly significant for $\beta_{p}(\psi)$ (a correction factor of $\sim 1.7$ to 1.9). The improved determinations of $\beta_{p}(\psi)$ and $D o L P_{p}(\psi)$ were validated with measurements on independent samples, and also using another independently-calibrated light scattering instrument, DAWN-EOS. Although the correction functions developed in this study are applicable only to the specific version of LISST-VSF instrument and the data processing code used in this study, our results emphasize a general need for evaluating the performance of light scattering instruments and minimizing the associated uncertainties in quantitative determinations from measurements. 

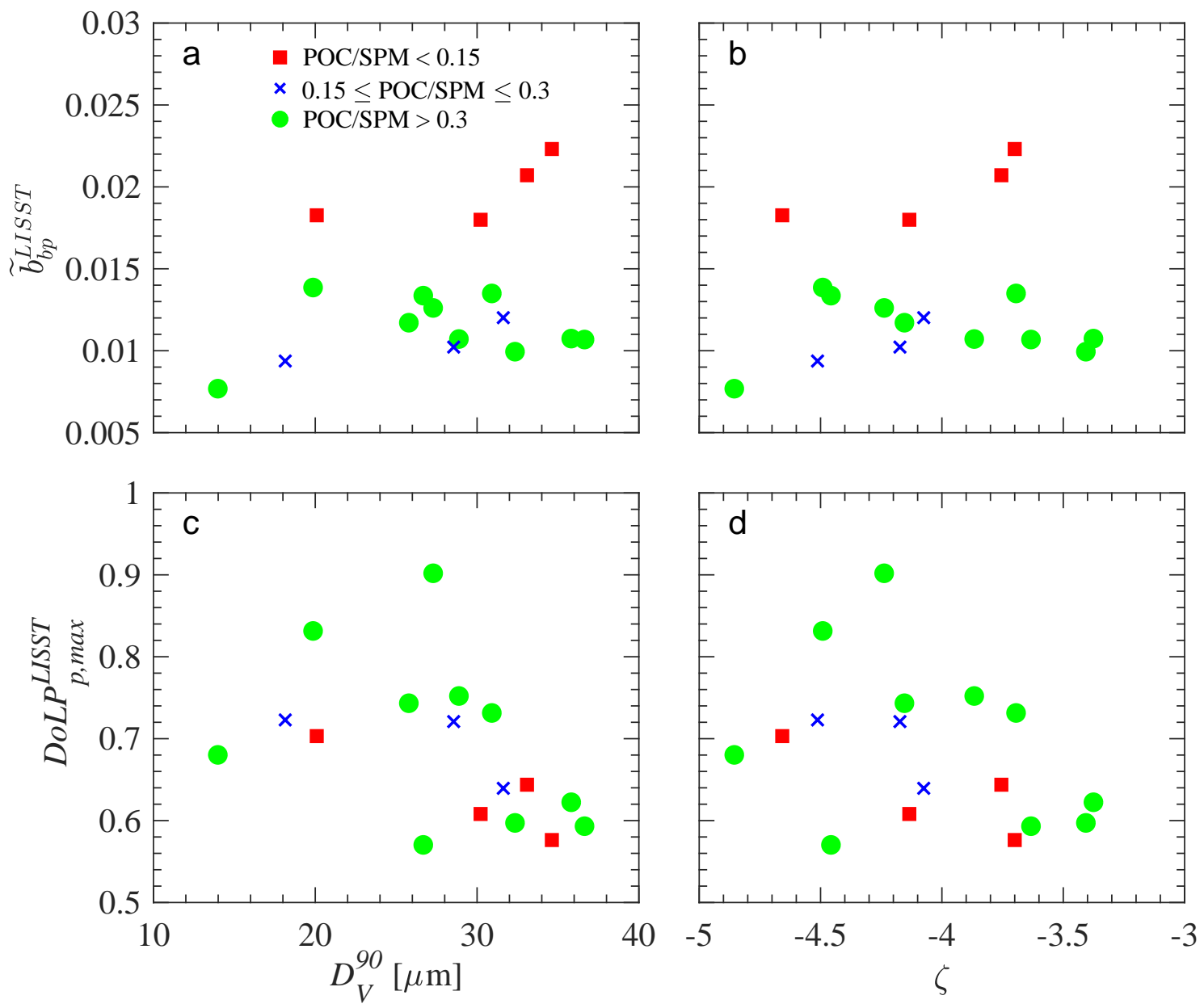

Figure 13. Similar to Figure 12, but with optical quantities shown as a function of the particle size metrics $(\mathbf{a}, \mathbf{c}) D_{V}^{90}$, representing the diameter corresponding to the $90^{\text {th }}$ percentile of the particle volume distribution, and $(\mathbf{b}, \mathbf{d}) \zeta$, the power law slope of the particle number distribution.

The improved protocol for measurements of light scattering with our LISST-VSF instrument was applied to measurements taken on 17 natural seawater samples from coastal and offshore marine environments characterized by contrasting assemblages of suspended particles. The particulate volume scattering function, degree of linear polarization, and backscattering ratio were determined from LISST-VSF measurements. For our dataset, these light scattering properties exhibit significant variations related to a broad range of measured particle properties characterizing the organic vs. inorganic composition and size distribution of particulate assemblages. For example, we observed negative relational trends between the particulate backscattering ratio and the increasing proportions of organic particles or phytoplankton in the total particulate assemblage. These proportions were parameterized in terms of the measured ratio of particulate organic carbon (POC) or chlorophyll- $a$ (Chla) concentration to the total dry mass concentration of suspended particulate matter (SPM). The observed trends can be useful in the development of optical approaches for characterizing the composition of particulate assemblages. Our results also suggest a potential trend between the maximum degree of linear polarization of light scattered by particles and particle size metrics. Specifically, the decrease in the maximum degree of linear polarization observed at scattering angles close to $90^{\circ}$ tends to be associated with particulate assemblages exhibiting a higher proportion of large-sized particles.

Earlier theoretical studies have shown that changes in the angular shape and the maximum value of the degree of linear polarization depend on particle refractive index and size distribution [82,83]; however, the experimental data of the degree of linear polarization of scattered light for natural marine 
particle assemblages are very scarce. Our results provide a contribution to filling this gap. This type of data can also be useful for improving an understanding of the polarization properties of marine light fields including polarization of water-leaving radiance and advancing related applications, including remote sensing applications [84-90]. The various potential applications of angular light scattering measurements, including the polarization effects associated with light scattering by marine particles, call for further efforts in this research area.

Author Contributions: All authors made intellectual contributions to the study design and participated in the collection of experimental data. D.K. and R.A.R. processed the data and conducted Mie scattering calculations, and D.K. analyzed the results. D.K. and D.S. wrote the paper, and all authors reviewed and provided final edits in the manuscript.

Acknowledgments: This work was supported by NASA Terrestrial Hydrology and Ocean Biology and Biogeochemistry Programs (Grant \#NNX13AN72G) and the NASA Earth and Space Science Fellowship Program (Grant \#NNX14AK93H). We acknowledge Edward R. Blocker, Eric Chen, and Linhai Li for assistance during measurements and processing of data. We also thank Xiaodong Zhang for providing computer code for backscattering extrapolation method and related discussions, and Wayne Slade for discussions about LISST-VSF instrument and processing code. We thank Hubert Loisel and three anonymous reviewers for valuable comments on the manuscript.

Conflicts of Interest: The authors declare no conflict of interest. The funding sponsors had no role in the design of the study; in the collection, analyses, or interpretation of data; in the writing of the manuscript, and in the decision to publish the results.

\section{References}

1. Kattawar, G.W.; Adams, C.N. Stokes vector calculations of the submarine light field in an atmosphere-ocean with scattering according to a Rayleigh phase matrix: Effect of interface refractive index on radiance and polarization. Limnol. Oceanogr. 1989, 34, 1453-1472. [CrossRef]

2. Mobley, C.D. Light and Water: Radiative Transfer in Natural Waters; Academic Press: San Diego, CA, USA, 1994.

3. Mobley, C.D.; Sundman, L.K.; Boss, E. Phase function effects on oceanic light fields. Appl. Opt. 2002, 41, 1035-1050. [CrossRef] [PubMed]

4. Kattawar, G.W.; Yang, P.; You, Y.; Bi, L.; Xie, Y.; Huang, X.; Hioki, S. Polarization of light in the atmosphere and ocean. In Light Scattering Reviews 10; Kokhanovsky, A.A., Ed.; Springer: Berlin, Germany, 2016; pp. 3-39.

5. Babin, M.; Morel, A.; Fournier-Sicre, V.; Fell, F.; Stramski, D. Light scattering properties of marine particles in coastal and open ocean waters as related to the particle mass concentration. Limnol. Oceanogr. 2003, 48, 843-859. [CrossRef]

6. Balch, W.M.; Gordon, R.G.; Bowler, B.C.; Drapeau, D.T.; Booth, E.S. Calcium carbonate measurements in the surface global ocean based on moderate-resolution imaging spectroradiometer data. J. Geophys. Res. 2005, 110, C07001. [CrossRef]

7. Stramski, D.; Reynolds, R.A.; Babin, M.; Kaczmarek, S.; Lewis, M.R.; Röttgers, R.; Sciandra, A.; Stramska, M.; Twardowski, M.S.; Franz, B.A.; et al. Relationships between the surface concentration of particulate organic carbon and optical properties in the eastern South Pacific and eastern Atlantic Oceans. Biogeosciences 2008, 5 , 171-201. [CrossRef]

8. Bale, A.J.; Morris, A.W. In situ measurement of particle size in estuarine waters. Estuar. Coast. Shelf Sci. 1987, 24, 253-263. [CrossRef]

9. Agrawal, Y.C.; Pottsmith, H.C. Instruments for particle size and settling velocity observations in sediment transport. Mar. Geol. 2000, 168, 89-114. [CrossRef]

10. Agrawal, Y.C.; Whitmire, A.; Mikkelsen, O.A.; Pottsmith, H.C. Light scattering by random shaped particles and consequences on measuring suspended sediments by Laser Diffraction. J. Geophys. Res. 2008, 113, C04023. [CrossRef]

11. Reynolds, R.A.; Stramski, D.; Wright, V.M.; Woźniak, S.B. Measurements and characterization of particle size distributions in coastal waters. J. Geophys. Res. 2010, 115, C08024. [CrossRef]

12. Wyatt, P.J.; Villalpando, D.N. High-precision measurement of submicrometer particle size distributions. Langmuir 1997, 13, 3913-3914. [CrossRef]

13. Wyatt, P.J. Submicrometer particle sizing by multiangle light scattering following fractionation. J. Colloid Interface Sci. 1998, 197, 9-20. [CrossRef] [PubMed] 
14. Uitz, J.; Stramski, D.; Baudoux, A.C.; Reynolds, R.A.; Wright, V.M.; Dubranna, J.; Azam, F. Variations in the optical properties of a particle suspension associated with viral infection of marine bacteria. Limnol. Oceanogr. 2010, 55, 2317-2330. [CrossRef]

15. Morel, A. Diffusion de la lumière par les eaux de mer: Resultats expérimentaux et approche théorique. In Optics of the Sea; North Atlantic Treaty Organization AGARD Lecture Series, No. 61; Technical Editing and Reproduction Ltd: London, UK, 1973; pp. 3.1.1-3.1.76.

16. Ackleson, S.G.; Spinrad, R.W. Size and refractive index of individual marine particulates: A flow cytometric approach. Appl. Opt. 1988, 27, 1270-1277. [CrossRef] [PubMed]

17. Twardowski, M.S.; Boss, E.; Macdonald, J.B.; Pegau, W.S.; Barnard, A.H.; Zaneveld, J.R.V. A model for estimating bulk refractive index from the optical backscattering ratio and the implications for understanding particle composition in case I and case II waters. J. Geophys. Res. 2001, 106, 14129-14142. [CrossRef]

18. Sullivan, J.M.; Twardowski, M.S.; Donaghay, P.L.; Freeman, S.A. Use of optical scattering to discriminate particle types in coastal waters. Appl. Opt. 2005, 44, 1667-1680. [CrossRef] [PubMed]

19. Zhang, X.; Huot, Y.; Gray, D.J.; Weidemann, A.; Rhea, W.J. Biogeochemical origins of particles obtained from the inversion of the volume scattering function and spectral absorption in coastal waters. Biogeosciences 2013, 10, 6029-6043. [CrossRef]

20. Bohren, C.F.; Huffman, D.R. Absorption and Scattering of Light by Small Particles; Wiley: New York, NY, USA, 1983.

21. Bickel, W.S.; Bailey, W.M. Stokes vectors, Mueller matrices, and polarized scattered light. Am. J. Phys. 1985, 53, 468-478. [CrossRef]

22. Kattawar, G.W. Polarization of light in the ocean. In Ocean Optics; Spinrad, R.W., Carder, K.L., Perry, M.J., Eds.; Oxford University Press: New York, NY, USA, 1994; p. 202.

23. Jonasz, M.; Fournier, G.R. Light Scattering by Particles in Water: Theoretical and Experimental Foundations; Academic Press: San Diego, CA, USA, 2007.

24. Fry, E.S; Voss, K.J. Measurement of the Mueller matrix for phytoplankton. Limnol. Oceanogr. 1985, 30, 1322-1326. [CrossRef]

25. Quinby-Hunt, M.S.; Hunt, A.J.; Lofftus, K.; Shapiro, D. Polarized-light scattering studies of marine Chlorella. Limnol. Oceanogr. 1989, 34, 1587-1600. [CrossRef]

26. Wyatt, P.J.; Jackson, C. Discrimination of phytoplankton via light-scattering properties. Limnol. Oceanogr. 1989, 34, 96-112. [CrossRef]

27. Shapiro, D.B.; Quinby-Hunt, M.S.; Hunt, A.J. Origin of the induced circular-polarization in the light scattered from a dinoflagellate. In Ocean Optics X; Spinrad, R.W., Ed.; SPIE: Bellingham, WA, USA, 1990; Volume 1302, pp. 281-289.

28. Witkowski, K.; Wolinski, L.; Turzynski, Z.; Gedziorowska, D.; Zielinski, A. The investigation of kinetic growth of Chlorella vulgaris cells by the method of integral and dynamic light-scattering. Limnol. Oceanogr. 1993, 38, 1365-1372. [CrossRef]

29. Volten, H.; De Haan, J.F.; Hovenier, J.W.; Schreurs, R.; Vassen, W.; Dekker, A.G.; Hoogenboom, H.J.; Charlton, F.; Wouts, R. Laboratory measurements of angular distributions of light scattered by phytoplankton and silt. Limnol. Oceanogr. 1998, 43, 1180-1197. [CrossRef]

30. Volten, H.; Muñoz, O.; Rol, E.; Haan, J.D.; Vassen, W.; Hovenier, J.W.; Nousiainen, T. Scattering matrices of mineral aerosol particles at $441.6 \mathrm{~nm}$ and $632.8 \mathrm{~nm}$. J. Geophys. Res. 2001, 106, 17375-17401. [CrossRef]

31. Volten, H.; Muñoz, O.; Hovenier, J.W.; Waters, L.B.F.M. An update of the Amsterdam light scattering database. J. Quant. Spectrosc. Radiat. Transf. 2006, 100, 437-443. [CrossRef]

32. Svensen, Ø.; Stamnes, J.J.; Kildemo, M.; Aas, L.M.S.; Erga, S.R.; Frette, Ø. Mueller matrix measurements of algae with different shape and size distributions. Appl. Opt. 2011, 50, 5149-5157. [CrossRef] [PubMed]

33. Muñoz, O.; Moreno, F.; Guirado, D.; Dabrowska, D.D.; Volten, H.; Hovenier, J.W. The Amsterdam-Granada light scattering database. J. Quant. Spectrosc. Radiat. Transf. 2012, 113, 565-574. [CrossRef]

34. Liu, J.P.; Kattawar, G.W. Detection of dinoflagellates by the light scattering properties of the chiral structure of their chromosomes. J. Quant. Spectrosc. Radiat. Transf. 2013, 131, 24-33. [CrossRef]

35. Stramski, D.; Boss, E.; Bogucki, D.; Voss, K.J. The role of seawater constituents in light backscattering in the ocean. Prog. Oceanogr. 2004, 61, 27-56. [CrossRef] 
36. Hovenier, J.W.; Volten, H.; Muñoz, O.; Van der Zande, W.J.; Waters, L.B.F.M. Laboratory studies of scattering matrices for randomly oriented particles: Potentials, problems, and perspectives. J. Quant. Spectrosc. Radiat. Transf. 2002, 79, 741-755. [CrossRef]

37. Kokhanovsky, A.A. Parameterization of the Mueller matrix of oceanic waters. J. Geophys. Res. 2003, $108,3175$. [CrossRef]

38. Petzold, T.J. Volume Scattering Functions for Selected Ocean Waters; SIO Ref. 72-78, Scripps Institution of Oceanography Visibility Lab; University of California: San Diego, CA, USA, 1972.

39. Beardsley, G.F. Mueller scattering matrix of sea water. J. Opt. Soc. Am. 1968, 58, 52-57. [CrossRef]

40. Kadyshevich, Y.A.; Lyubovtseva, Y.S.; Rozenberg, G.V. Light-scattering matrices of Pacific and Atlantic ocean waters. Izv. Acad. Sci. USSR Atmos. Ocean. Phys. 1976, 12, 106-111.

41. Kadyshevich, Y.A. Light-scattering matrices of inshore waters of the Baltic Sea. Izv. Acad. Sci. USSR Atmos. Ocean. Phys. 1977, 13, 77-78.

42. Voss, K.J.; Fry, E.S. Measurement of the Mueller matrix for ocean water. Appl. Opt. 1984, 23, 4427-4439. [CrossRef] [PubMed]

43. Lee, M.; Lewis, M. A new method for the measurement of the optical volume scattering function in the upper ocean. J. Atmos. Ocean. Technol. 2003, 20, 563-572. [CrossRef]

44. Sullivan, J.M.; Twardowski, M.S. Angular shape of the oceanic particulate volume scattering function in the backward direction. Appl. Opt. 2009, 48, 6811-6819. [CrossRef] [PubMed]

45. Tan, H.; Doerffer, R.; Oishi, T.; Tanaka, A. A new approach to measure the volume scattering function. Opt. Express 2013, 21, 18697-18711. [CrossRef] [PubMed]

46. Chami, M.; Thirouard, A.; Harmel, T. POLVSM (Polarized Volume Scattering Meter) instrument: An innovative device to measure the directional and polarized scattering properties of hydrosols. Opt. Express 2014, 22, 26403-26428. [CrossRef] [PubMed]

47. Zhang, X.; Lewis, M.; Lee, M.; Johnson, B.; Korotaev, G. The volume scattering function of natural bubble populations. Limnol. Oceanogr. 2002, 47, 1273-1282. [CrossRef]

48. Twardowski, M.S.; Zhang, X.; Vagle, S.; Sullivan, J.; Freeman, S.; Czerski, H.; You, Y.; Bi, L.; Kattawar, G. The optical volume scattering function in a surf zone inverted to derive sediment and bubble particle subpopulations. J. Geophys. Res. 2012, 117, C00H17. [CrossRef]

49. Slade, W.H.; Agrawal, Y.C.; Mikkelsen, O.A. Comparison of measured and theoretical scattering and polarization properties of narrow size range irregular sediment particles. In Oceans San Diego; IEEE: San Diego, CA, USA, 2013; pp. 1-6.

50. Slade, W.H.; Boss, E.S. Calibrated near-forward volume scattering function obtained from the LISST particle sizer. Opt. Express 2006, 14, 3602-3615. [CrossRef] [PubMed]

51. Van de Hulst, H.C. Light Scattering by Small Particles; Dover Publications: New York, NY, USA, 1981.

52. McCartney, E.J. Optics of the Atmosphere: Scattering by Molecules and Particles; Wiley: New York, NY, USA, 1976.

53. Mishchenko, M.I.; Travis, L.D. Light scattering by polydisperse, rotationally symmetric nonspherical particles: Linear polarization. J. Quant. Spectrosc. Radiat. Transf. 1994, 51, 759-778. [CrossRef]

54. Yanamandra-Fisher, P.A.; Hanner, M.S. Optical properties of nonspherical particles of size comparable to the wavelength of light: Application to comet dust. Icarus 1999, 138, 107-128. [CrossRef]

55. Petrova, E.V.; Jockers, K.; Kiselev, N.N. Light scattering by aggregates with sizes comparable to the wavelength: An application to cometary dust. Icarus 2000, 148, 526-536. [CrossRef]

56. Muñoz, O.; Volten, H.; Hovenier, J.W.; Min, M.; Shkuratov, Y.G.; Jalava, J.P.; van der Zande, W.J.; Waters, L.B.F.M. Experimental and computational study of light scattering by irregular particles with extreme refractive indices: Hematite and rutile. Astron. Astrophys. 2006, 446, 525-535. [CrossRef]

57. Muinonen, K.; Zubko, E.; Tyynelä, J.; Shkuratov, Y.G.; Videen, G. Light scattering by Gaussian random particles with discrete-dipole approximation. J. Quant. Spectrosc. Radiat. Transf. 2007, 106, 360-377. [CrossRef]

58. Zubko, E. Light scattering by irregularly shaped particles with sizes comparable to the wavelength. In Light Scattering Reviews 6; Kokhanovsky, A.A., Ed.; Springer: Berlin, Germany, 2012; pp. 39-74.

59. Agrawal, Y.C. The optical volume scattering function: Temporal and vertical variability in the water column off the New Jersey coast. Limnol. Oceanogr. 2005, 50, 1787-1794. [CrossRef]

60. Agrawal, Y.C.; Mikkelsen, O.A. Empirical forward scattering phase functions from 0.08 to 16 deg. for randomly shaped terrigenous 1-21 $\mu \mathrm{m}$ sediment grains. Opt. Express 2009, 17, 8805-8814. [CrossRef] [PubMed] 
61. Babin, M.; Stramski, D.; Reynolds, R.A.; Wright, V.M.; Leymarie, E. Determination of the volume scattering function of aqueous particle suspensions with a laboratory multi-angle light scattering instrument. Appl. Opt. 2012, 51, 3853-3873. [CrossRef] [PubMed]

62. Heller, W.; Tabibian, R.M. Experimental investigations on the light scattering of colloidal spheres. II. Sources of error in turbidity measurements. J. Colloid Sci. 1957, 12, 25-39. [CrossRef]

63. Bateman, J.B.; Weneck, E.J.; Eshler, D.C. Determination of particle size and concentration from spectrophotometric transmission. J. Colloid Sci. 1959, 14, 308-329. [CrossRef]

64. Stramski, D.; Babin, M.; Woźniak, S.B. Variations in the optical properties of terrigenous mineral-rich particulate matter suspended in seawater. Limnol. Oceanogr. 2007, 52, 2418-2433. [CrossRef]

65. Woźniak, S.B.; Stramski, D.; Stramska, M.; Reynolds, R.A.; Wright, V.M.; Miksic, E.Y.; Cieplak, A.M. Optical variability of seawater in relation to particle concentration, composition, and size distribution in the nearshore marine environment at Imperial Beach, California. J. Geophys. Res. 2010, 115, C08027. [CrossRef]

66. Bohren, C.F. Multiple scattering of light and some of its observable consequences. Am. J. Phys. 1987, 55, 524-533. [CrossRef]

67. LISST-VSF Multi-Angle Polarized Light Scattering Meter: User'S Manual Revision A; Sequoia Scientific: Bellevue, WA, USA.

68. Ma, X.; Lu, J.Q.; Brock, R.S.; Jacobs, K.M.; Yang, P.; Hu, X.H. Determination of complex refractive index of polystyrene microspheres from 370 to $1610 \mathrm{~nm}$. Phys. Med. Biol. 2003, 48, 4165-4172. [CrossRef] [PubMed]

69. Morel, A.; Bricaud, A. Inherent optical properties of algal cells including picoplankton: Theoretical and experimental results. Can. Bull. Fish. Aquat. Sci. 1986, 214, 521-559.

70. Van der Linde, D.W. Protocol for determination of total suspended matter in oceans and coastal zones. JRC Tech. Note I 1998, 98, 182.

71. Parsons, T.R.; Maita, Y.; Lalli, C.M. A Manual of Chemical and Biological Methods for Seawater Analysis; Elsevier: New York, NY, USA, 1984.

72. Knap, A.; Michaels, A.; Close, A.; Ducklow, H.; Dickson, A. Protocols for the Joint Global Ocean Flux Study (JGOFS) Core Measurements; UNESCO: Paris, France, 1994.

73. Ritchie, R.J. Universal chlorophyll equations for estimating chlorophylls a, b, c, and d and total chlorophylls in natural assemblages of photosynthetic organisms using acetone, methanol, or ethanol solvents. Photosynthetica 2008, 46, 115-126. [CrossRef]

74. Bader, H. The hyperbolic distribution of particle sizes. J. Geophys. Res. 1970, 75, 2822-2830. [CrossRef]

75. Beardsley, G.F., Jr.; Zaneveld, J.R.V. Theoretical dependence of the near-asymptotic apparent optical properties on the inherent optical properties of sea water. J. Opt. Soc. Am. 1969, 58, 373-377. [CrossRef]

76. Zhang, X.; Fournier, G.R.; Gray, D.J. Interpretation of scattering by oceanic particles around 120 degrees and its implication in ocean color studies. Opt. Express 2017, 25, A191-A199. [CrossRef] [PubMed]

77. Zhang, X.; Hu, L.; He, M. Scattering by pure seawater: Effect of salinity. Opt. Express 2009, 17, 5698-5710. [CrossRef] [PubMed]

78. Reynolds, R.A.; Stramski, D.; Neukermans, G. Optical backscattering by particles in Arctic seawater and relationships to particle mass concentration, size distribution, and bulk composition. Limnol. Oceanogr. 2016, 61, 1869-1890. [CrossRef]

79. Ivanoff, A. Optical method of investigation of the oceans: The p- $\beta$ diagram. J. Opt. Soc. Am. 1959, 49, $103-104$. [CrossRef]

80. Ivanoff, A.; Jerlov, N.; Waterman, T.H. A comparative study of irradiance, beam transmittance and scattering in the sea near Bermuda. Limnol. Oceanogr. 1961, 6, 129-148. [CrossRef]

81. Hatch, T.; Choate, S.P. Measurement of polarization of the Tyndall beam of aqueous suspension as an aid in determining particle size. J. Franklin Inst. 1930, 210, 793-804. [CrossRef]

82. Chami, M.; Santer, R.; Dilligeard, E. Radiative transfer model for the computation of radiance and polarization in an ocean-atmosphere system: Polarization properties of suspended matter for remote sensing. Appl. Opt. 2001, 40, 2398-2416. [CrossRef] [PubMed]

83. Lotsberg, J.K.; Stamnes, J.J. Impact of particulate oceanic composition on the radiance and polarization of underwater and backscattered light. Opt. Express 2010, 18, 10432-10445. [CrossRef] [PubMed]

84. Waterman, T.H. Polarization patterns in submarine illumination. Science 1954, 120, 927-932. [CrossRef] [PubMed] 
85. Ivanoff, A. Polarization measurements in the sea. In Optical Aspects of Oceanography; Jerlov, N.G., Steeman-Nielsen, E., Eds.; Academic Press: London, UK; New York, NY, USA, 1974; pp. 151-175.

86. Chami, M. Importance of the polarization in the retrieval of oceanic constituents from the remote sensing reflectance. J. Geophys. Res. 2007, 112, C05026. [CrossRef]

87. Loisel, H.; Duforet, L.; Dessailly, D.; Chami, M.; Dubuisson, P. Investigation of the variations in the water leaving polarized reflectance from the POLDER satellite data over two biogeochemical contrasted oceanic areas. Opt. Express 2008, 16, 12905-12918. [CrossRef] [PubMed]

88. Tonizzo, A.; Gilerson, A.; Harmel, T.; Ibrahim, A.; Chowdhary, J.; Gross, B.; Ahmed, S. Estimating particle composition and size distribution from polarized water-leaving radiance. Appl. Opt. 2011, 50, 5047-5058. [CrossRef]

89. Ibrahim, A.; Gilerson, A.; Chowdhary, J.; Ahmed, S. Retrieval of macro- and micro-physical properties of oceanic hydrosols from polarimetric observations. Rem. Sens. Environ. 2016, 186, 548-566. [CrossRef]

90. Zhai, P.W.; Knobelspiesse, K.; Ibrahim, A.; Franz, B.A.; Hu, Y.; Gao, M.; Frouin, R. Water-leaving contribution to polarized radiation field over ocean. Opt. Express 2017, 25, A689-A708. [CrossRef] [PubMed]

(C) 2018 by the authors. Licensee MDPI, Basel, Switzerland. This article is an open access article distributed under the terms and conditions of the Creative Commons Attribution (CC BY) license (http:/ / creativecommons.org/licenses/by/4.0/). 